\title{
Functional Architecture of Spatial Attention in the Parietal Cortex of the Behaving Monkey
}

\author{
Milena Raffi and Ralph M. Siegel \\ Center for Molecular and Behavioral Neuroscience, Rutgers University, Newark, New Jersey 07102
}

\begin{abstract}
Functional architectures facilitate orderly transmittal of representations between cortices, allow for local interactions between neurons, and ensure a uniform distribution of feature representations with respect to larger-scale topographies. We sought to correlate such topographies with internal cognitive states. A psychophysical task for which the monkey was required to detect a change in one of two identical peripheral expanding flow fields tested for spatial shifts of attention. The monkey was cued as to which flow would change with a small cue near the fixation points. Reaction time data indicate that the monkey's performance in the optic flow detection task depended on the location of the cue. Using optical imaging of intrinsic signals, we show that a monkey's internally generated locus of attention is correlated with an $800-860 \mu \mathrm{m}$ patchy topological architecture across the cortical surface of the inferior parietal lobule. The attentional patches vary in location but are stable in spatial frequency. The patches are embedded in a larger-scale and stable representation of eye position. Trial-by-trial analysis of the images indicates that the organizational scheme with simultaneous stable and variable subcomponents occurs within the experiment of $1 \mathrm{~d}$, as well as across days. This novel functional architecture is the first to be correlated with attentional mechanisms and could support a fine-scale functional architecture underlying hemispatial neglect, an attentional deficit caused by parietal lesions.
\end{abstract}

Key words: cortical topography; eye position; gain field; extrastriate visual cortex; optical imaging; macaque

\section{Introduction}

Single-unit recordings in the inferior parietal lobule of behaving monkeys are correlated with eye position, oculomotor, visual, and attentional signals (Bushnell et al., 1981; Motter and Mountcastle, 1981; Mountcastle et al., 1981; Andersen et al., 1985, 1990; Read and Siegel, 1997; Constantinidis and Steinmetz, 2001). However, electrophysiological studies have not located any topographical order across the cortical surface to these processes because of the impossibility of making reconstructions over the years needed to complete such studies.

Changes in eye position alter the strength of individual neuron responses to visual stimuli in the parietal cortex (Andersen et al., 1985; Read and Siegel, 1997). Optical imaging of changes in the oxygenation of hemoglobin within and across the cortical surface permits the evaluation of global neuronal network activity (Malonek and Grinvald, 1996). Area 7a and the dorsal prelunate (DP) area, two gyral portions of the inferior parietal lobule, are accessible to direct visualization for intrinsic optical imaging (Siegel et al., 2003). Area 7a, which lies close to the intraparietal sulcus, is modulated by the inferior, contralateral position of the

Received June 10, 2004; revised April 13, 2005; accepted April 14, 2005.

This work was supported by National Institutes of Health Grants R01 EY-09223, R03 DA011687, and 1S10RR12873 and by National Science Foundation Grant NPACI RUT223. The massive file transfer and storage services provided by the Storage Resource Broker team at the San Diego Supercomputer Center are acknowledged.

Correspondence should be addressed to Dr. Ralph M. Siegel, Center for Molecular and Behavioral Neuroscience, Rutgers University, 197 University Avenue, Newark, NJ 07102. E-mail: axon@cortex.rutgers.edu.

M. Raffi's present address: Department of Human and General Physiology, University of Bologna, Piazza di Porta

S. Donato 2, 40126 Bologna, Italy. E-mail: milena@biocfarm.unibo.it.

DOI:10.1523/JNEUROSCI.5201-04.2005

Copyright $\odot 2005$ Society for Neuroscience $\quad$ 0270-6474/05/255171-16\$15.00/0 eye in the orbit. The neighboring DP represents the superior, contralateral eye position. A push-pull relationship between these two areas suggests that they may have a distributed representation of the position of the eye (Siegel et al., 2003).

Single-unit studies have also shown parietal neurons are selective to the retinotopic location of a visual stimulus (Andersen et al., 1985; Read and Siegel, 1997). Optical imaging confirms this element of spatial perception in the behaving monkey in a unexpected manner (Heider et al., 2005). Reproducible retinotopic maps were obtained daily in the dorsalmost aspect of DP (DPd). Within each of two monkeys, this representation within DPd was unchanging across months of observation. In contrast, the representation of upper and lower retinotopy in area 7a was not consistent across the days of recording, confirming the suggestion (Gilbert, 1993) that cortical topographic maps can change. We sought to determine whether the variability seen in area $7 \mathrm{a}$ was the result of ongoing covert attentional or other cognitive mechanisms that were not explicitly under experimental control during these procedures.

First, we demonstrated that there is a distribution of the tuning of neurons for spatial attention across areas $7 \mathrm{a}$ and dorsal prelunate gyrus. The approach was to have the monkeys perform an adaptation of a task long recognized to demonstrate shifts of spatial attention (Posner, 1980). Because area 7a neurons respond strongly to optic flow stimuli (Siegel and Read, 1997), we had the monkey respond rapidly to one of two identical optic flow stimuli. A visual cue was used to direct the monkeys' "attentional spotlight" (Crick, 1984) to the location of a specific change in optic flow of one of the stimuli. Intrinsic optical imaging was then performed on the visualized cortex during the task. A 
"patchy" representation of attention was embedded within the eye position gain field map. The role of this embedded representation in guiding attention and in hemispatial neglect is considered.

\section{Materials and Methods}

All experiments were approved by the Rutgers University Institutional Animal Care and Use Committee and were performed according to the guidelines published by the National Institutes of Health.

Surgical procedures. Experiments were performed in two rhesus monkeys $(6.5-7.5 \mathrm{~kg})$. All surgical procedures were identical to those described previously (Siegel et al., 2003; Heider et al., 2005); these two monkeys were used in the previous work and can be identified by their naming codes. Briefly, the monkeys were prepared for chronic experiments with emphasis on exceptional head rigidity for repeated optical measurements. A $20 \mathrm{~mm}$ chamber was placed over the right hemisphere in the first monkey (M1R) and the left hemisphere in the second monkey (M2L), with a chronically implanted artificial dura permitting long-term optical recordings (Shtoyerman et al., 2000; Siegel et al., 2003; Heider et al., 2005).

Behavioral task. Monkeys performed a reaction-time task based on a standard spatial attention paradigm (Posner, 1980; Posner and Petersen, 1990) in which its attention is cued to one of two spatially separate yet identical stimuli. In any one experimental run, the task was performed with two eye positions (e.g., $10^{\circ}$ above or below the primary position) (Fig. 1a). After the onset of the $0.1^{\circ}$ red fixation point, the monkey pulled a lever and began fixation. After $2000 \mathrm{~ms}$, two identical expanding optic flow stimuli were presented $10^{\circ}$ on either side of the red fixation point, at right angles to the line connecting the two fixation point locations (e.g., $10^{\circ}$ to the right or left) (Fig. 1a). Simultaneously, a $1^{\circ}$ diameter cue was presented $2.5^{\circ}$ on one side of the fixation point, to instruct the monkey which nearby flow stimulus would later change. The monkey had to detect a change from structured to unstructured (i.e., random) (Siegel and Andersen, 1988) in the cued optic flow within a reaction time window. The cued change in the expansion optic flow occurred at a time randomly selected in the time window of $4000-5500 \mathrm{~ms}$ after the trial began and had to be detected within a reaction time window of $150-800 \mathrm{~ms}$. Correct fixation and key releases were rewarded with juice; this task was performed $90-100 \%$ correct.

In one set of experiments, two vertical eye positions were used. In a second set of experiments run on different days, two horizontal eye positions ( $10^{\circ}$ to the left and right of the primary position) were used. In the experiments when the fixation positions were up and down, the flow stimuli were presented to the left and right, with the cue being to the left or right. In the experiments when the fixation positions were left and right, the optic flow stimuli were above and below the fixation point; the cue was either above or below. Larger cue eccentricities ( 5 and $10^{\circ}$ ) were used on experimental days when the monkey's performance was poor for the smaller eccentricities ( $26 \%$ of 115 experiments). The occasional poor performance was attributed to the animal's motivation.

To determine whether the monkey had indeed attended to the cued optic flow stimulus, a behavioral experiment was run in which $20 \%$ of the trials contained an invalid cue, i.e., the monkey was cued to attend to one optic flow stimulus, but the change in the structure occurred in the other one (Fig. 1b); typically, the "invalid" trials should have a longer reaction time (Posner, 1980). The behavioral control experiments were run approximately once per week to avoid the monkeys becoming aware of the invalid cue and altering their strategy. The monkeys had a consistent behavioral strategy of attending to the cued optic flow stimulus. Figure $1 d$ illustrates the data from a single behavioral test from M1R; the invalid cue significantly increased the monkey's reaction time in all four conditions (ANOVA; $p<0.05$ ).

All of the dots forming the optic flow stimulus moved uniformly at 6 $\%$ s radially from the center of expansion. The stimulus consisted of 48 $0.1^{\circ}$ white points $\left(10^{\circ}\right.$ in diameter); their centers were displaced $10^{\circ}$ from the fixation point on either side. The point life was $533 \mathrm{~ms}$, and the dots flickered asynchronously. Initially, the fraction of structure was 1; after the change to the unstructured motion, the fraction of structure was 0 (Siegel and Andersen, 1988). (The stimulus looked like randomly moving flickering dots when the fraction of structure was 0 .) The $1^{\circ}$ radius cue stimulus was typically positioned $2.5^{\circ}$ (and sometimes $10^{\circ}$ ) from the red fixation point and was made of 32 dots flickering with $533 \mathrm{~ms}$ point life.

All tasks were performed with the head fixed and eye position monitored with an ISCAN (Cambridge, MA) infrared tracker. Incorrect key release or eye movements $>1^{\circ}$ terminated the trial.

Behavioral data were analyzed by an ANOVA of the reaction time taken for the key to be released relative to the instant the motion started to change from structured to unstructured optical flow. A three-way ANOVA was computed with "cue validity" (whether the cue position correctly predicted optic flow change), "cue position," and "eye position" as the independent terms (PROC GLM; SAS Institute, Cary, NC); all interaction effects were permitted. The probability of the main or interaction effects were tallied on a run-by-run basis. Typically, the monkey performed from 400 to 800 correct trials on any given day; hence, there were 100-200 correct trials per condition. Cue validity was taken as significant when there was an interaction or independent effect at $p<0.05$.

The task was designed to shift the monkeys' attention to one location, 
hold it there, and avoid inhibition of return (Posner, 1980; Taylor and Klein, 2000). In the classical studies on the inhibition of return, subjects responded to a task in which the cue was flashed in a peripheral field, followed by a variable delay, followed by the stimulus onset. The inhibition of return occurs when the attention is removed from a peripheral location, so that there is a delay in responding to a stimulus presented in that portion of visual field. In our task, the cue was always present together with the stimulus, and the monkeys did not have to shift their attention toward a new location; they had to keep their attention on the optic flow stimulus throughout the task. Hence, inhibition of return should be minimized in these behavioral studies.

Optical imaging. The inferior parietal lobule was optically studied, because it has been implicated by spatial attention in single-unit electrophysiological experiments (Bushnell et al., 1981; Mountcastle et al., 1981; Constantinidis and Steinmetz, 2001). Optical imaging data from two surface areas, $7 \mathrm{a}$ and DP, were collected while the monkeys performed an experiment of a fixed block design of two eye positions by two cued stimulus locations. The designation DP will be used here to refer to the dorsalmost aspect of this gyrus and corresponds to DPd as used by Heider et al. (2005). Imaging was performed with an Optical Imaging (Rehovot, Israel) VDAQ 2001 system (Siegel et al., 2003; Heider et al., 2005). The cortex was illuminated at $605 \mathrm{~nm}$ via a filtered halogen bulb powered by stabilized direct current (DC) supply; frames were collected at $7 \mathrm{~Hz}$. Thirty-seven frames were collected starting at the fixation point onset. The camera was focused at a depth of $500 \mu \mathrm{m}$ below the surface capillaries. Images were collected at $\sim 720 \times 480$ pixel resolution and binned online to $\sim 360 \times 240$ pixels. Simultaneously, behavioral data were collected by a second computer. All data were moved to an IBM SP2 for combination and analysis. All behavioral error trials were eliminated from the data set. The experiments were performed for 14 months in monkey M1R and for 5 months in M2L. A total of 170 maps were collected: 124 in M1R and 46 in M2L. The experiments were performed at a range of magnifications (23-54 pixels/mm); the features reported here were magnification invariant.

Data analysis. One hundred to 200 correct trials per condition (400800 total) were collected. Data was converted from the Optical Imaging format and analyzed with the Khoral Research (Albuquerque, NM) package in conjunction with SAS. As in previous work (Siegel et al., 2003), the "difference signal" on a trial-by-trial basis was computed from the activity at $2000-3000 \mathrm{~ms}$ after stimulus onset after subtraction of the baseline signal $[-1000,0 \mathrm{~ms}]$ relative to stimulus onset. These times are when the visually evoked optical signal peaks (Siegel et al., 2003; Heider et al., 2005). This difference was normalized by the baseline signal to give a percentage change. Trial rejection was performed on masked data. A mask to obliterate large surface blood vessels was computed, and the average cortical signal excluding the masked regions was computed for every trial. Trials that were $>1 \mathrm{SD}$ of the mean of all trials were excluded from analysis to remove outliers (Siegel et al., 2003). This procedure is termed the baseline normalization analysis (BNA) by Siegel et al. (2003).

All additional analysis was performed on all of the pixels in the images. The sign of the signals were inverted to account for the expectation that the optical signal at $605 \mathrm{~nm}$ is inverted from expected electrical activity (Malonek and Grinvald, 1996; Siegel et al., 2003). As per Siegel et al. (2003), no filtering was applied to the data.

The optical signal for pixel $(I, J)$ was modeled with two different models. Equation 1 had a linear sum and interaction of eye position in degrees $\left(E_{i}\right)$ and the cued stimulus position in degrees $\left(\varphi_{i}\right)$, to match with the behavioral conditions. Equation 2 did not have the interaction term. These models were given as follows:

$O_{i}(I, J)=A(I, J) E_{i}+B(I, J) \varphi_{i}+C(I, J) E_{i} \varphi_{i}+D(I, J)+\varepsilon_{i}(I, J)$,

or

$$
O_{i}(I, J)=A(I, J) E_{i}+B(I, J) \varphi_{i}+D(I, J)+\varepsilon_{i}(I, J),
$$

where $O_{i}$ is the optical signal for the $i$ th trial, $A$ is the eye position coefficient, $B$ is the cue position coefficient, $D$ is the intercept, and $\varepsilon_{i}$ is the residual error. An interaction between eye position and cue location was modeled by the term $C$. [The indices $(I, J)$ are dropped for clarity.] Both models were computed, and the Akaike Information Criterion (AIC) (Akaike, 1969) was used to choose the better model for each pixel. Overall, the purely linear model (Eq. 2 ) was a better model for $84.5 \pm 4.9 \%$ of the pixels in 10 experiments examined; thus, the purely linear model was used to model the optical signal across all pixels. An example of the selection of the model using the AIC analysis for the data of Figure 2 is provided as Figure 3. It should be noted that the noise in the data, as well as any effects not accounted for by the model, were accounted for by the residual error term $\varepsilon_{i}$.

The purely linear model (Eq. 2) defines a planar surface in eye and cued stimulus position. The intercept $D$ represents the interpolated signal for both the eye position and the cue position at the origin $\left(E_{i}=\varphi_{i}=0\right)$. The $A$ and $B$ are the steepness of the slope for the eye and cue dependency. To simultaneously represent these two dependencies, a circle space representation was constructed to represent the experimental conditions resulting in the greatest signal modulation (i.e., steepest slope). The angle of the plane for each pixel is given as $\phi(I, J)=\arctan [B(I, J) / A(I, J)]$. Thus, $\phi=(0, \pi)$ would be a pure eye position tuning, whereas $\phi=(-\pi / 2, \pi / 2)$ would represent a pixel modulated only by the cue location and, hence, the locus of attention. The magnitude of the slope in that direction was given as the length of the vector $(A, B)$ from the origin.

A three-step procedure estimated the spatial frequency and phase components of parameter maps and single trials. First, a $512 \times 512$ floating point image was constructed with a value of 0 everywhere as the digital fast Fourier transform (FFT) required power of images with dimension of power of 2 . The $370 \times 240$ pixel image under consideration was embedded in the center of this image. Because the FFT is periodic, the sharp boundaries induce a spurious spatial frequency. This is reduced by multiplying the image by a circular Gaussian mask (maximum amplitude, 1; spatial coefficient, 77 pixels); second, an FFT was then used to compute a complex value for each horizontal and vertical frequency combination. For each of these points, the complex numbers were converted to a power and phase, resulting in two spatial frequency maps. The third step was to reduce the two-dimensional FFT to one dimension. A series of 18 cuts in frequency space (every $10^{\circ}$ ) were made through the power component of the FFT, each going through the origin. Each cut yielded a one-dimensional FFT for power as a function of spatial frequency. Some of these would have low power because the original image had low power in that orientation, whereas others would have greater power. The power under each of these one-dimensional FFTs was computed, and the one-dimensional FFT with the greatest power was selected as a model of the data. The phase as a function of spatial frequency for this orientation was also recorded. These procedures are similar to those of Obermayer and Blasdel (1993).

\section{Results}

\section{Psychophysics of behavioral task}

To determine whether the monkeys' attention was directed to the cued optic flow stimulus, a behavioral experiment was run in which $20 \%$ of the trials contained an invalid cue (Fig. 1b); typically, the invalid trials should have a longer reaction time (Posner, 1980). The behavioral control experiments were run approximately once per week to avoid the monkeys becoming aware of the invalid cue and altering their strategy. The monkeys had a consistent behavioral strategy of attending to the cued optic flow stimulus. Figure $1 d$ illustrates the data from a single behavioral test from M1R; the invalid cue significantly increased the monkey's reaction time in all four conditions (ANOVA, $p<0.05$ ). In 23 of $32(72 \%)$ behavioral tests in the two monkeys, there was a significant effect of the validity of the cue on reducing the reaction time, demonstrating the effect of the attentional spotlight. However, this was most often seen as an additive or interaction effect with the eye position in 17 of 23 experiments (Fig. 1e).

A stepwise linear regression for the dependence of the reaction 
time on the eye position and cue position was performed for both animals. The model was given as a linear sum plus an interaction term for eye position and cue position. Using the stepwise model, forty-six experiments demonstrated significant effects in the linear model; of these, 31 experiments had significant effects $(p<0.05)$ for a dependence on eye position and/or cued flow position with no interaction effect. The remaining 15 had interaction effects. (These interaction effects are complicated to examine graphically and are not described further.) The eye position/ attentional locus effect was examined by animal to illustrate their strategy (Fig. 4). A point is provided for each experiment for which purely linear effects of eye position and/or attentional locus were found. An average vector was computed across all such experiments for each animal. Individually, each animal demonstrated a vector significantly different from the origin $\left(0^{\circ}, 0^{\circ}\right)(\mathrm{M} 1 \mathrm{R}, p<0.0001, n=23$; M2L, $p=0.02, n=8$; Hotelling's one-sample test) (Batschelet, 1981). The two populations of reaction time dependencies were significantly different $(p=0.012$, Watson-Williams two-sample test) (Batschelet, 1981), indicating that the two monkeys had significantly different performances.

For example, M1R had a mean vector formed from the slope for eye position dependence and the slope of cue position dependence of $\left(0.82 \mathrm{~ms} /{ }^{\circ}, 0.30 \mathrm{~ms} /{ }^{\circ}\right)$. This indicates that, on average, a $10^{\circ}$ downward shift of eye position increased the reaction time by $\sim 8.2$ ms with a right shift of the focus of attention position increasing the reaction time by $3.0 \mathrm{~ms}$. Combining these parameters, the longest increase in reaction time would be $8.73 \mathrm{~ms}$ for a downward eye position and a rightward shift of attention; conversely, the largest decrease in reaction time would be for an upward eye position and a leftward attentional target of $8.73 \mathrm{~ms}$. For M2R, the corresponding values would be -3.0 and $15.4 \mathrm{~ms}$, corresponding to a $15.7 \mathrm{~ms}$ maximal change. It can be noted that the effects on any one day can be much greater than these aggregate averages, with some days having effects as large as $40 \mathrm{~ms}$ for a $10^{\circ}$ shift in attention or eye position.

This dependence on eye position as well as cue validity indicates that a particular eye position may alter the attentional processing mechanisms or that the animal has particular biases. Behavioral biases in monkeys are not unique; there is a retinotopic bias for motion perception (Newsome et al., 1985), as well as for oculomotor tasks (Wardak et al., 2002; Schiller and Tehovnik, 2003). In our study, these biases were not "trained" into the animal but appear to represent some strategy that develops unconstrained by the task. Surprisingly, to the best of our knowledge, interactions between eye and cue position have not been studied with psychophysics in human or nonhuman primates. If such biases are confirmed in other studies, this suggests interplay in their representation in the cortex
Cued Stimulus Task
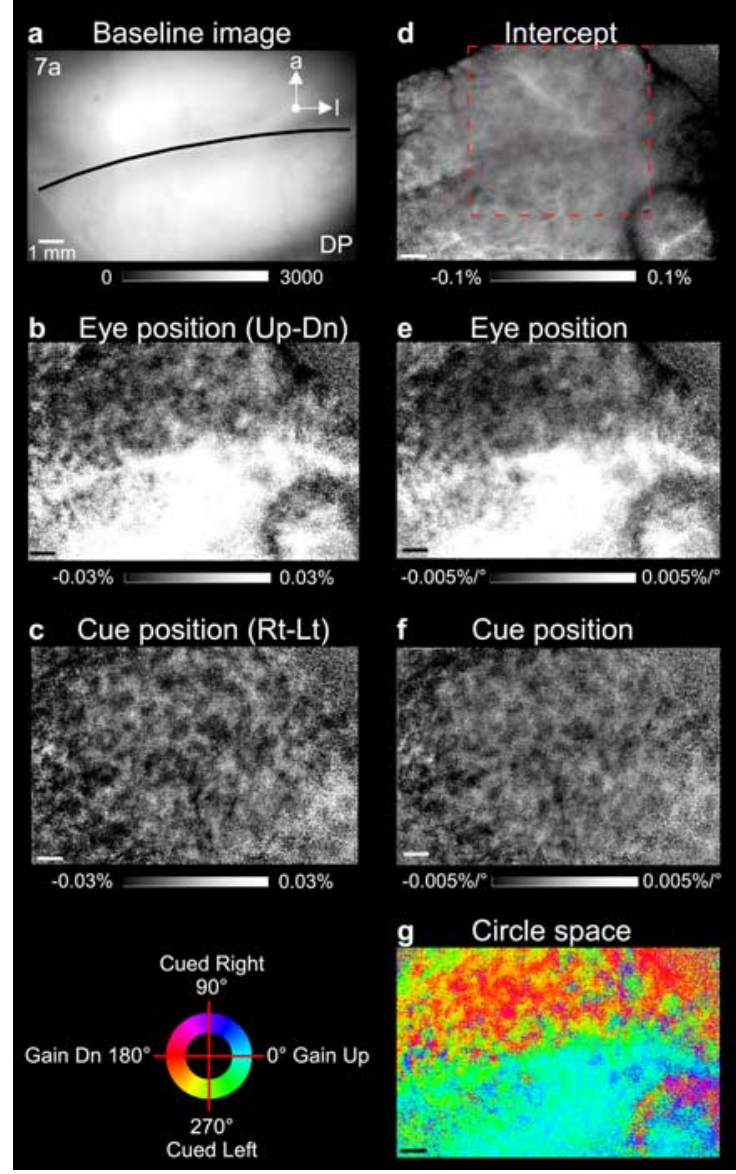

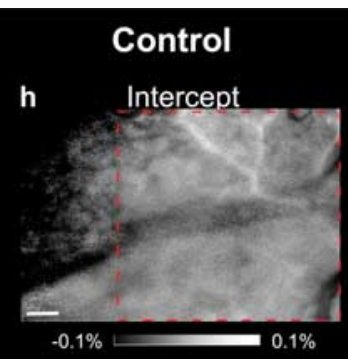

i Eye position
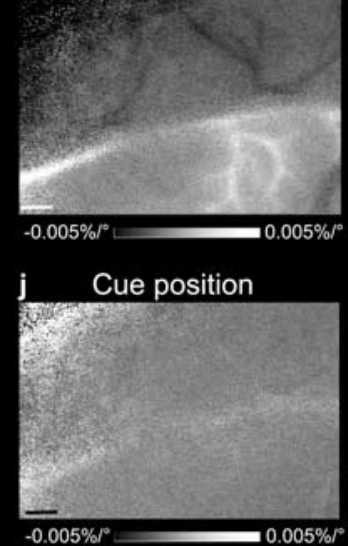

k Circle space

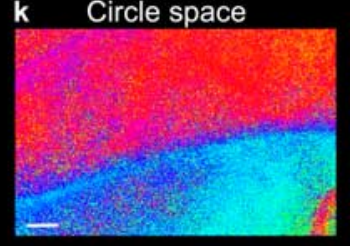

Figure 2. Cortical topography for spatial attention in the inferior parietal lobule. $\boldsymbol{a}$, Average reflected light across the cortex imaged with red $(605 \mathrm{~nm}$ ) illumination. The red light masks most of the blood vessel and permits imaging of deoxyvalues from the 0 l system. The black line shows the putative border between the two areas based on a large vessel running subtracted from all downward (Dn) fixation conditions. c, Subtraction cued stimulus position map; leftward (Lt) conditions were subtracted from rightward (Rt) cued conditions. A linear regression analysis was used to quantify these The red dashed boxes in $\boldsymbol{d}$ and $\boldsymbol{h}$ show the regions common to both experiments. All data are from animal M1R (a-g, 04-05-2001/gm; $\boldsymbol{h}-\boldsymbol{k}, 10-05-2001 / \mathrm{gm})$. a, Anterior;l, lateral. The chamber edge can be seen at the bottom left. Note that the translucent silicon ring at the bottom left edge of the chamber functions as a conduit to the reflected light, providing a distorted image of the underlying cortex. Scale bars, $1 \mathrm{~mm}$.

\section{Optical recordings}

Optical cortical responses to upward versus downward fixations verified previous results on the topography of gain fields in areas $7 \mathrm{a}$ and DP; indeed, the data matched that from the same hemispheres studied previously (Siegel et al., 2003). A difference of the average of the images collected when the animal fixated in the two different vertical positions showed the appropriate increases and decreases in reflected $605 \mathrm{~nm}$ light (Fig. $2 b$ ). The darkness apparent across the extent of area $7 \mathrm{a}$ indicated that it was activated by lower field fixations, and the lightness across the extent of area DP indicated activation by upper visual fields. However, a new feature was observed never seen in the previous studies of these animals: patchy regions with a scale of $\sim 0.5-1.5 \mathrm{~mm}$ superimposed on the gain fields. This feature appeared in the measurement for which the rightward cued image was differenced from 


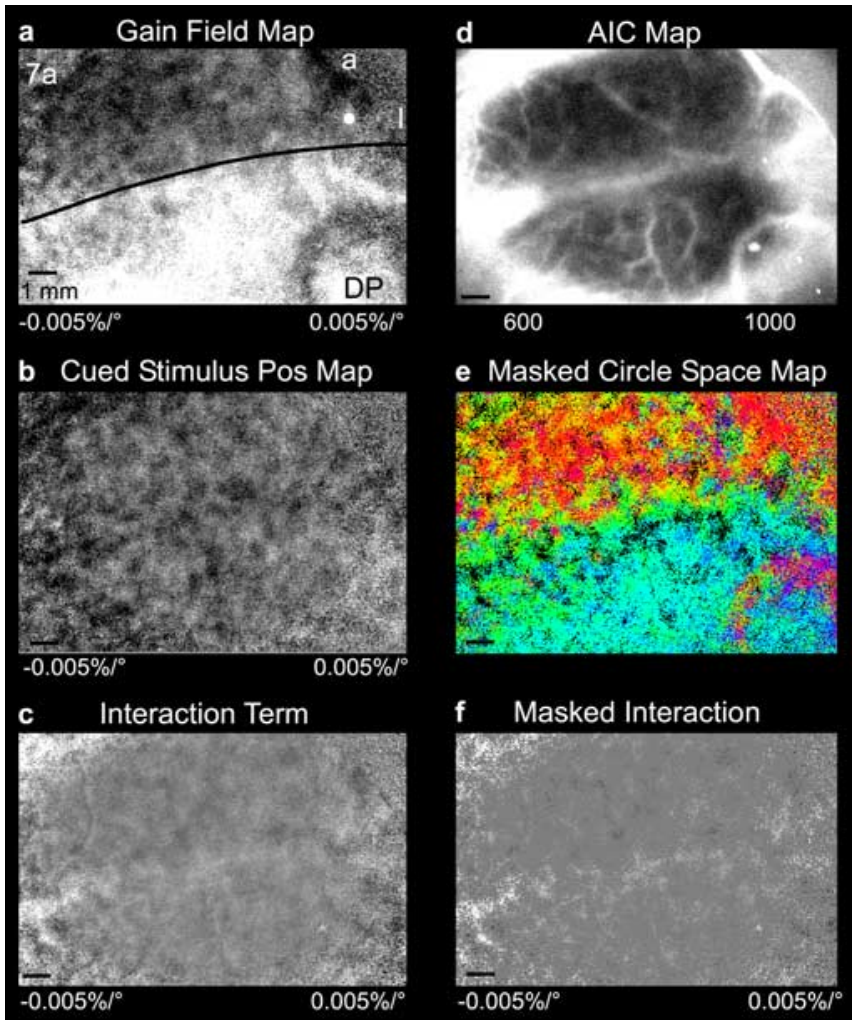

Figure 3. Use of AIC to select model for regression. Two models, one with (Eq. 1) and one without (Eq. 2) an interaction term were used to model the change in reflected light (see Materials and Methods). The parameters were eye position (gain parameter) and the position of the stimulus cue. The resulting parameter maps of the interaction model (Eq. 1) are as follows: $\boldsymbol{a}$, eye position parameter; $\boldsymbol{b}$, stimulus cue; $\boldsymbol{c}$, interaction term. $\boldsymbol{d}$, The AIC was separately computed for each pixel for each equation. The AIC map for the interaction model (Eq. 1) is shown. The AIC map for the linear model looked the same as that for the linear with interaction model. However, when the AIC was compared on a pixel-by-pixel basis between the two models, almostall of the pixels in the model without the interaction (Eq. 2) had a lower value. The results for the model without the interaction are shown in Figure 2. e, Masked circle space map. Pixels are masked black for the map in which the AIC indicated that the model with interaction was better. $\boldsymbol{f}$, Masked interaction map. Similarly, the pixels in the interaction parameter map were set to 0 (middle gray) for the pixels that were best modeled by the linear model. The range for the gray scale for each image is indicated below each image. The circle space convention is the same as in Figure 2. All data are from animal M1R (04-05-2001/gm). Pos, Position. Scale bars, $1 \mathrm{~mm}$.

the left cued image (Fig. 2c). These patches could represent the topographical distribution of electrophysiological attentional effects (Bushnell et al., 1981; Mountcastle et al., 1981; Constantinidis and Steinmetz, 2001).

The contribution of the eye position and cue effects were first quantified using a linear regression model allowing for two additive terms to account for the variance in the optical signal (see Materials and Methods). Three effects were associated with the task. First, the intercept (Fig. $2 d$ ) was found to be independent of eye and cue position. Second, the gain field parameter map indicated an eye-dependent modulation, replicating the previous result (Siegel et al., 2003) of area 7a representing the lower visual field and DP the upper visual field (Fig. 2e). Third, a cued stimulus location map was found that was clearly patchy (Fig. $2 f$ ). This last map suggested a fine spatial structure for the horizontal loci of attention (Fig. $2 f$ ).

The possibility that the cued stimulus position and the gain field signals interact, as might be expected from electrophysiological recordings (Andersen et al., 1985, 1990; Read and Siegel, 1997), was examined by a comparison of models (see Materials

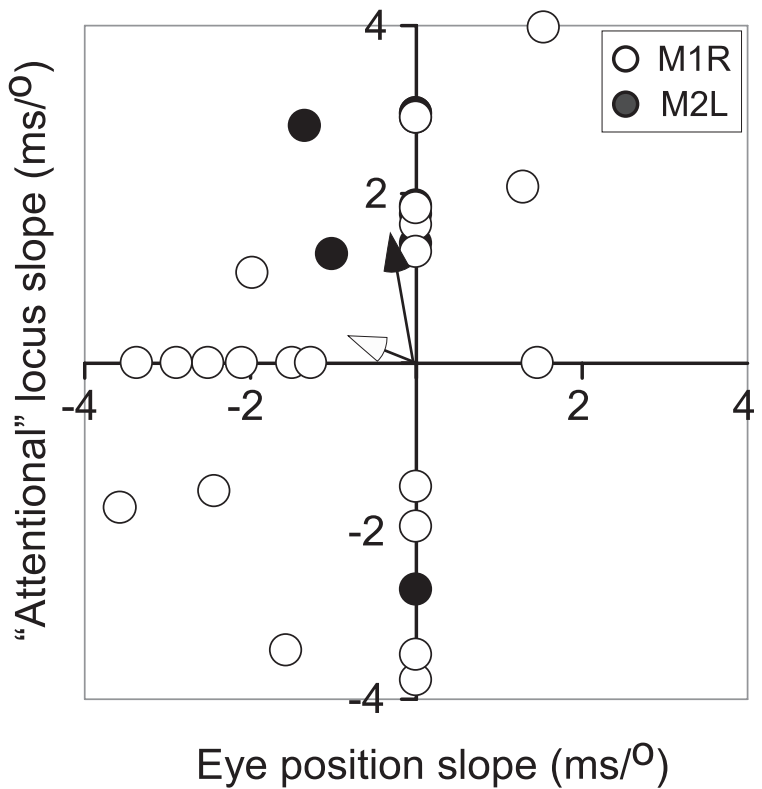

Figure 4. Behavioral dependence of reaction time on eye position and cue position. The behavioral experiments for which eye position was up-down and the cue position was leftright were analyzed. For each experiment, the reaction time was regressed as a function of eye position and cue position: $\mathrm{RT}_{i}=a E_{i}+b \phi_{i}+c E_{i} \phi_{i}+d+\varepsilon_{i}$, where $a$-dare regression coefficients $\varepsilon_{i}$ is the trial error for the ith trial and RT is the reaction time. A stepwise regression (Read and Siegel, 1997) was used to ensure that all coefficients were significantly different from 0 at $p<0.05$. For those 31 experiments for which only $a$ and/or $b$ were significant and $c$ was not significant, a vector was formed $(a, b)$; these are graphed here. The average vector by animal was computed, and circular statistics showed that each animal's average performance was significantly different from the origin and from each other (Batschelet, 1981). The average behavioral dependence for each animal is shown by the drawn line.

and Methods and Fig. 3) (Heider et al., 2005). It was not possible to fully separate the data with patches into a "spatially flat" gain field and a fully patchy cued position map. The addition of an interaction term (Eq. 1) was not a better model for the data according to the AIC, and higher-order models were not attempted to better separate the effects. Although there are higher-order models that could be used, typically, the AIC has a single minimum, suggesting that we had reached the best model for the data. More complex models could have been used, but the small signalto-noise ratio of the intrinsic signal precludes such testing. It is noted that it is unlikely that the patches emerged simply from the use of regression models, because previous studies (Siegel et al., 2003; Heider et al., 2005) using very similar models in the same animals never showed patches of the sort described herein. In conclusion, using numerical approaches, within the framework of the data we have, it was not possible to improve on the model with separable eye position and cue position maps.

The overall signal of more reflectance in DP and less in area $7 \mathrm{a}$ fits with the previous study (Siegel et al., 2003). That there were patches in the eye position-dependent map was unexpected in that vertical gain fields are reasonably flat (Siegel et al., 2003). A color circle space representation combining the tuning of each pixel to the eye position and the cued stimulus position illustrates that both the horizontal position of the cued stimulus location and the eye position was systematically represented across the crown of the inferior parietal lobule (Fig. $2 g$ ). The broad shift in colors between 7a and DP represents previously established gain field tuning, whereas the patches represent the cue positiondependent tuning. 


\section{Time course of the optical signal}

The time course of the tuning was examined in multiple ways. First, individual patches were selected by drawing regions of interest "by eye" over different colored portions of the map computed from the difference images. The average signals for each of these square regions of interests were then computed on a frame-by-frame basis. The baseline was used to normalize the signal (Fig. 5). Eleven such patches demonstrate that the time course of the signal for a rightward cue is strongly modulated by eye position (Fig. $5 G$, compare the two solid lines). Similarly, the response to the leftward cue is modulated by eye position (compare the dashed lines). Comparison of the thick solid to the thick dotted lines illustrates the effect of the cue position (and presumably attentional focus). The magnitude and direction of each of these effects varies with position on the cortex. The time course of each of these patches can be seen to depend on both the eye position and the location of cue that directed the monkey's "attention." The relative amplitudes of each of these time courses matches that expected using the difference in the baseline versus evoked signal (i.e., baseline normalization analysis).

The selection of patches, although often obvious, does have an element of arbitrariness. A second technique to examine the time course was to segment the maps based on the sign of regression coefficients. Rather than extract all of the individual patches, the cortical map was segmented into four groups, each representative of the possible combinations of two eye positions and two cue positions. To do this, each regression coefficient map was segmented into two portions: all positive and all negative values. All logical combinations of these resulted in four exclusive segmentations: the portion of the image with positive eye position gain and positive cue $(\mathrm{E}+\mathrm{C}+)$, positive eye, the portion with negative cue $(\mathrm{E}+\mathrm{C}-)$, etc. The time course for each of these four segments was thus computed by masking the original time course data with each of these four segmentations. Figure $6 a$ illustrates the original map, with Figure $6 b$ showing the four segments or masks. The four time courses corresponding to the four stimulus conditions are shown for each segmented region (Fig. 6c). For example, in the top right part of $b$, much of the masked region corresponds to area DP. In the top right part of $c$, the blue lines are above the black lines, corresponding to more reflected light with lower eye positions. This is as expected because DP represents upper eye position (recall that the reflected light is inversely related to the expected single-unit signals). The separation between each pair of dotted and solid lines indicates the effect of the cue position (and most probably attention). The other masked regions of interest have different tuning. To summarize these effects, the average response from the two yellow shaded regions are computed from the time course and differences computed in a similar manner to baseline normalization analysis. The relative tuning of the differ-
A
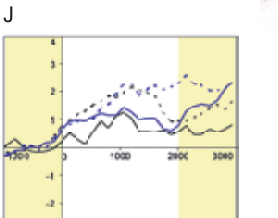

B
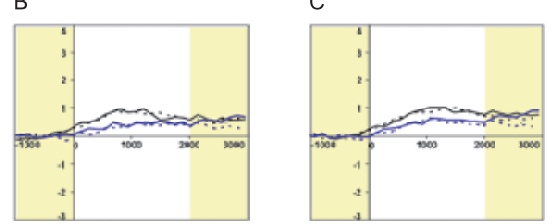

D
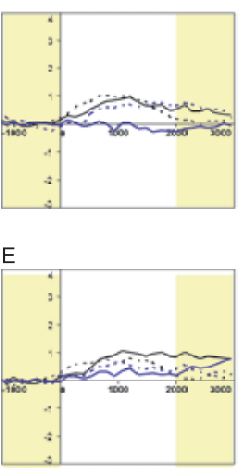

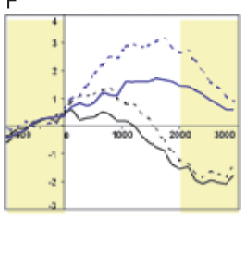

G

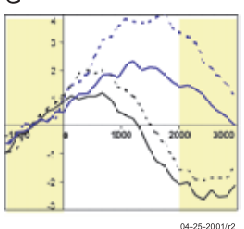

Figure 5. Time dependence of optical signal for selected patches of cortex. The color map at the center of the figure was used the ect square regions of interest. The color key is depicted to the left. The time course for each region of interest was plotted for uses the change in signal over the two yellow-shaded regions. The vertical axis in each graph is the reflected light normalized by

\section{(1)}

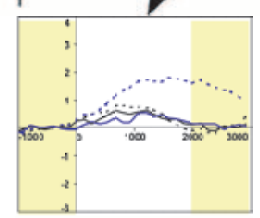

H

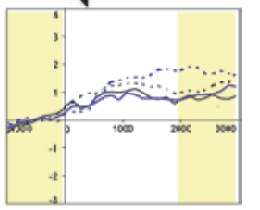

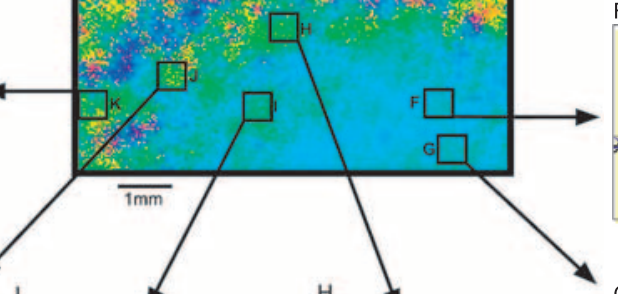

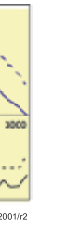


a
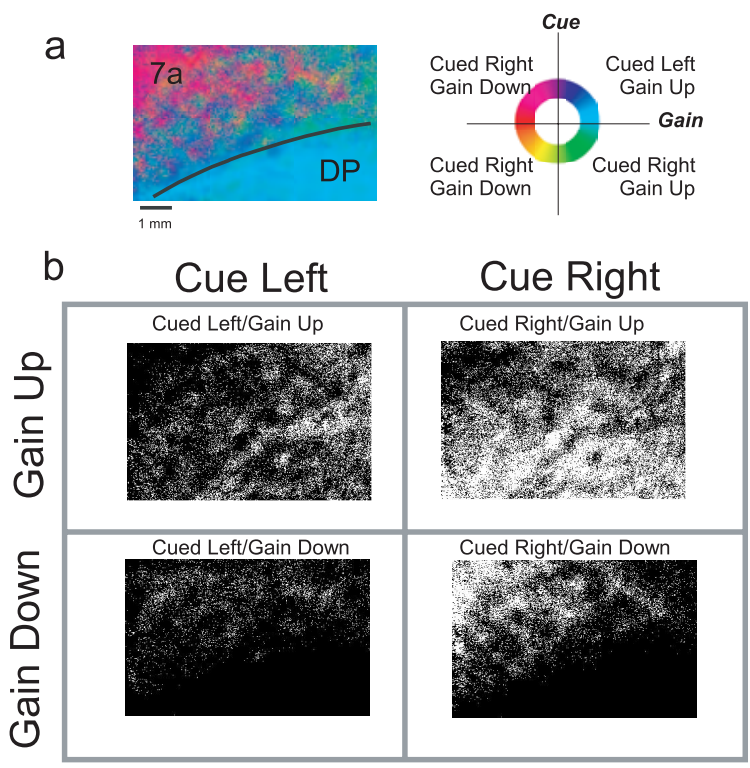

c Cue Left

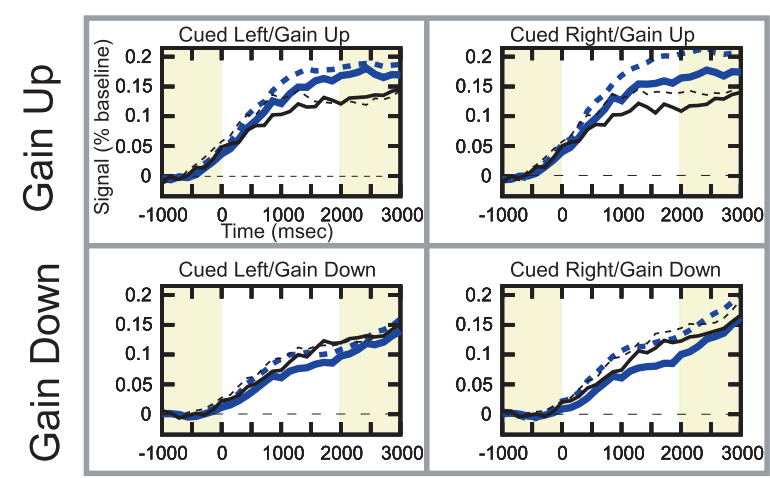

d
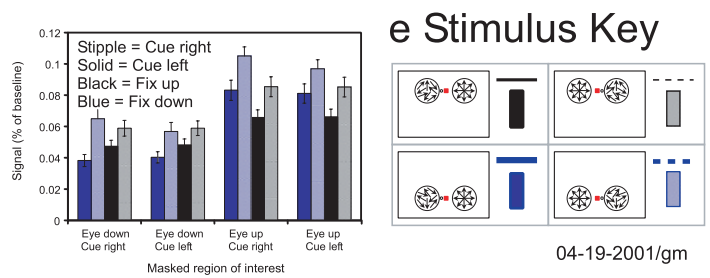

Figure 6. Time dependence of optical signal for cortex segmented by gain and cue parameter maps. $\boldsymbol{a}$, Gain and cue position map. This map was formed using the BNA and linear regressions. The color key is to the right. $\boldsymbol{b}$, Four masks were computed for each of the four possible combinations of eye (up and down) by cue (left and right). Each of these masks corresponds to one quadrant of the color key of $\boldsymbol{a}$. This "Cue Left/Gain Up" mask is the set of pixels for lies between 0 and $90^{\circ}$ in the color key (predominantly blue quadrant). $c$, Time course of the optical signal for each of the masked regions. To compute this time course, the average response for each of the four stimulus conditions was computed on a frame-by-frame basis. This provided $\sim 80,000$ time courses, each with 37 frames for each of the four stimulus conditions. The pixels for each stimulus condition were then averaged using the four masks of $\boldsymbol{b}$. This yielded 16 time courses of 37 frames each. Hence, the top left graph of c is the average time course computed for the pixels of the corresponding mask of $\boldsymbol{b}$. The four lines in the graph correspond to each of the four stimulus conditions. For this graph, there is a clear separation between the blue and black lines that arises from the effect of eye position on the response. The differences in the dashed and solid lines represent the effect of the cue on the time course for those masked pixels. In the top right (Cued Right/Gain Up) of c, the effect of the cue position is stronger than for the "Cued Left/Gain Up" masked data. $d$, The average response over the yellow-shaded regions is computed, and the difference is taken for the baseline $[-1000,0]$ versus evoked $[2000,3000 \mathrm{~ms}]$ data and presented as a bar graph. The values are grouped by the masked regions of interest. The SEs are computed across the 14 values in each shaded region.e, Stimulus key is provided for $\mathbf{c}$ and $\boldsymbol{d}$. (Data are from 04-19-2001/gm.) dent on the eye position topographic representation than previously realized. Indeed, two human case studies indicate that eye position-dependent attentional neglect can occur (Nadeau and Heilman, 1991; Nadeau et al., 1997). As suggested by Heilman, the eye position dependence of attentional signals may permit another affordance toward rehabilitation for patients with parietal neglect.

\section{Control for presence of cue stimulus}

The patches in the cued stimulus position map had two possible basis; first, the cue could directly activate the cortex as a "sensory" stimulus (Motter and Mountcastle, 1981; Andersen et al., 1990), and, second, it could cause a spatial shift in the locus of attention, thus altering the cortical topography. The former is unlikely because area 7a and DP neurons are essentially "blind" to small unattended stimuli (Motter and Mountcastle, 1981; Andersen et al., 1990). Even so, the effects of the small stimuli were tested on the optical response. A control experiment was performed with the cue presented in the absence of the two optic flow stimuli; the attention was directed to the fixation point by the requirement of the monkeys' detection of its slight dimming, i.e., the eccentric cue had no relevance to the completion of the task (Fig. 1c). Thus, the task sequence was the fixation point came on at one of two locations; $2000 \mathrm{~ms}$ later, the small test cue came on to the right or left. At a time randomly selected over the interval of 5000-6000 $\mathrm{ms}$ after the fixation onset, the fixation point dimmed. If the animal released the key within the reaction time window, he received a juice reward. Thus, the monkey was required to attend to the central red fixation point and ignore the cue.

The patches were not observed in the cue and eye position maps when the cue was behaviorally irrelevant, yet the gain field effect could still be observed (Fig. $2 h-k$ ). Thus, the presence of the cue alone has no measurable direct sensory effect on these intrinsic optical signals.

\section{Control for presentation of a pair of optic flow fields}

Although there were no patches for the cue-only condition compared with the standard task, it is possible that the patches could simply arise from the presence of the two flow fields. As a control for this, one of the two monkeys (M1R) performed a task for which no cue was present. Thus, the animal was required to fixate, but he did not know which of the two displays was to change. (In these experiments, the flow was presented above and below the fixation point. As shown in Figures 15 and 16, cued attention up and down under similar conditions leads to patches.) Under these conditions ( $n=8$ experiments), the patches were not found in the difference maps when the monkeys were fixating straight ahead (Fig. 7). Under these conditions, the monkey had two possible strategies. First, he could spread his attentional focus broadly to encompass both displays. Alternatively, he could shift attention back and forth between the two displays. During the period of optical data collection and analysis, the monkey saw identical visual stimulation and did not know which of the two stimuli were to change. In some experiments, the monkey showed a bias to one side. For the 23 psychophysical tests, six showed a significant effect of reaction time at $p<0.05$; of these, five showed a leftward bias and one showed a rightward bias.

It was also of interest to compare the mean reaction time for this task with the cued optic flow task. If the monkey was indeed shifting his attention or distributing his attention between the two flow fields in the control task in which two flows were presented in the absence of the cue, the overall reaction time would be expected to be longer. Data was compared for tasks in which 


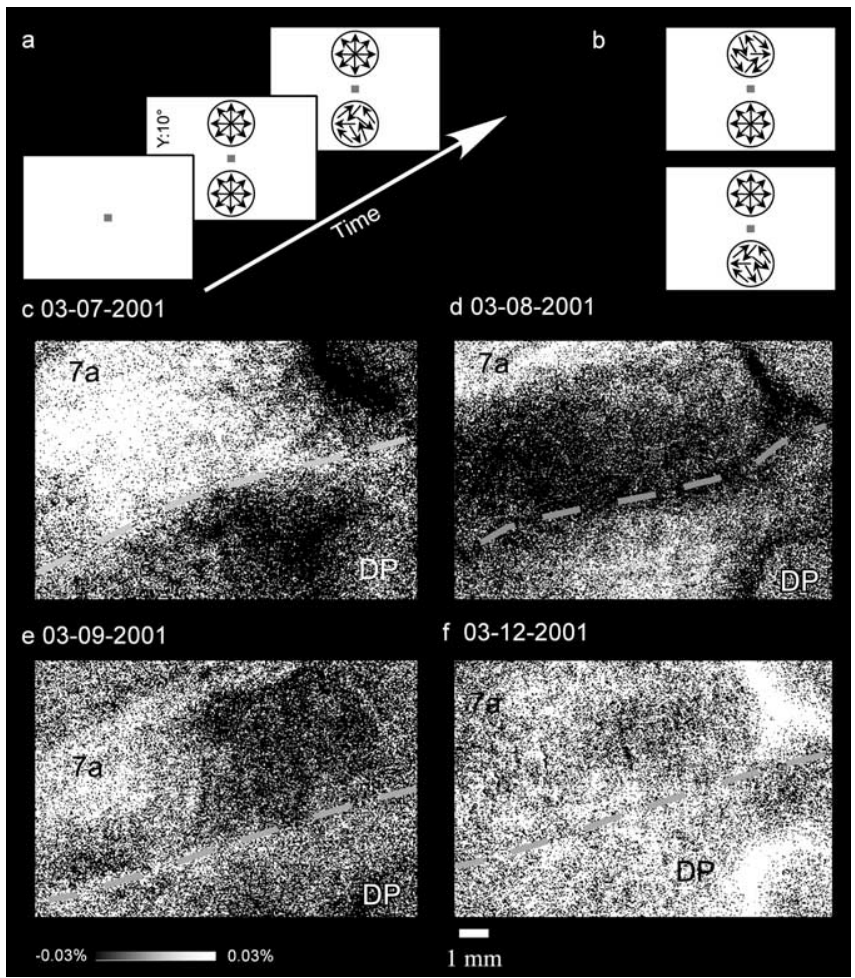

Figure 7. Simultaneous presentation of two flow fields in the absence of a cue. $\boldsymbol{a}, \mathrm{M} 1 \mathrm{R}$ performed a task for which a red fixation point was presented at the primary position $\left(0^{\circ}, 0^{\circ}\right)$ followed at $2000 \mathrm{~ms}$ by a pair of flow fields. A cue as to which flow field was to change was never given. At a random interval $2000-3500$ ms later, one of the two changed, and the animal released the key for a juice reward. The timing for evaluating the change in the optical signal was as described in Materials and Methods for the other experiment. Thus, the stimulus configuration during the optical measurement was exactly the same in the two conditions. $\boldsymbol{b}$, The two stimulus configurations used. In one, the upper flow field changes, and, in the other, the lower field changed. c, Four examples of the difference maps. The average response from the trials when the upper stimulus changed was subtracted from the trials when the lower stimulus changed.

the cue was above or below the fixation point. The reaction times were $404.6 \pm 107.8 \mathrm{~ms}$ for the standard cued task $(n=2744$ trials) and $433.3 \pm 120.2 \mathrm{~ms}$ for the uncued two-flow control described in this section $(n=2641)$. These two groups were significantly different at $p<0.001$. As expected, the control task in which only the cue was presented and the animal needed to respond to a dimmed fixation point had the shortest reaction time of $377.6 \pm 41.3$ ( $n=1242$ trials). (The large number of trials was obtained by grouping across experiments for each of the different test conditions.)

These behavioral and optical results indicate that the presence of the two optic flow stimuli cannot solely account for the patches and that the animal did not have an upper versus lower preference for the stimulus position when he was not cued for position. Furthermore, it can be concluded from the two controls conditions that the patches occur only when the animals are shifting their attention to one of the two identical flow fields. The regression analysis suggests that patches occur correlated with the cues; the presence of the patches in the eye position parameter maps suggests that there is a finer-scale modulation of the gain fields over the broad modulation between area $7 \mathrm{a}$ and DP reported previously (Siegel et al., 2003).

\section{Patch quantification using spatial fast Fourier transforms}

The patches in the attentional task are qualitatively $\sim 1 \mathrm{~mm}$ in size. In the control task, they appeared to be absent. A numerical a Cue position parameter map

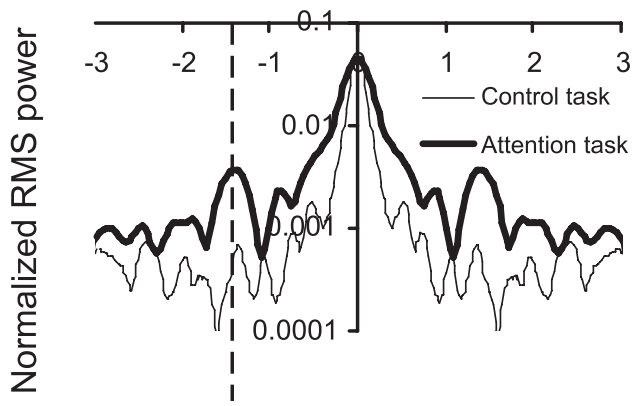

b Eye position parameter map

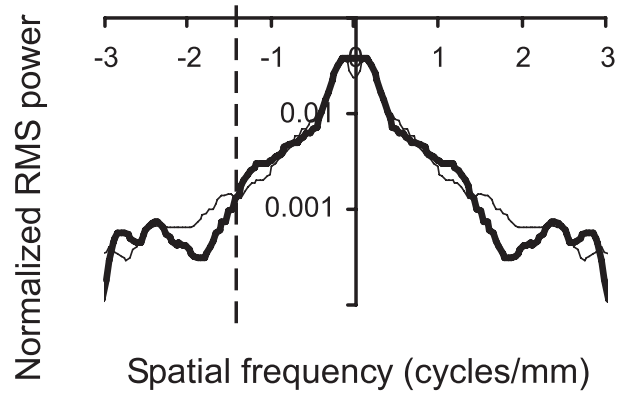

Figure 8. Spatial frequency analysis of parameter maps collected with the spatial attention task and the control task. $\boldsymbol{a}$, Spatial frequency dependence of the power for the cue parameter map. The attentional task (thick line) and the control task (thin line) have been normalized to have the same total power. Peaks at 1.47 cycles/mm corresponding to a spatial wavelength of $680 \mu \mathrm{m}$ are found in the attentional task. Each power spectrum was normalized by the total root mean square power, which was 0.250 and $0.116 \% / \% / \mathrm{mm}$ for the attentional and control task, respectively. $\boldsymbol{b}$, Spatial frequency dependence for the gain field parameter map. Conventions are as in $\boldsymbol{a}$. There was little periodic modulation of gain field signals in either case. The total power was 0.677 and $0.480 \% / \% \mathrm{~mm}$ for the attentional and control experiments. The data are from M1R, 04-05-2001/gm and 10-05-2001/gm for the attentional and control task, respectively (i.e., from Fig. 2).

calculation termed the "spatial FFT" was used to quantify the spatial frequency content of the parameter maps (see Materials and Methods). It is similar in concept to the well known timebased fast Fourier transform that is often used to compute the temporal frequencies inherent in a time-varying sequence.

The FFTs of the parameter maps for the attentional cued and the control experiments were computed; two examples are shown in Figure 8. Two experiments were selected that imaged similar regions of cortex at the same magnification. Under the attention cued experiments, there was a clear peak at $1.47 \mathrm{cy}-$ cles $/ \mathrm{mm}$ that corresponds to a characteristic spatial wavelength of $680 \mu \mathrm{m}$ (Fig. $8 a$, thick line). By comparison, the normalized root mean square (RMS) power of the control experiment (Fig. $8 a$, thin line) was always less than that of the attentional experiment. Indeed, at the spatial wavelength of $680 \mu \mathrm{m}$, the normalized RMS power of the control task was 11.9-fold less than that of the attentional task. This analysis of two experiments suggests that the scale of the patches is $680 \mu \mathrm{m}$ and that they are absent when the monkey performs the control task. The gain fields for the two experimental conditions were extremely similar (Fig. 8b), indicating matched low-frequency components of this eye position-related signal. Indeed, although FFT analysis was not performed, $\sim 1 \mathrm{~mm}$ patches were never evident by inspection in the original gain field studies in the inferior parietal lobule (Siegel et al., 2003).

These results were consistent across experiments, as shown by averaging the RMS power of the FFTs (independent of phase) for 

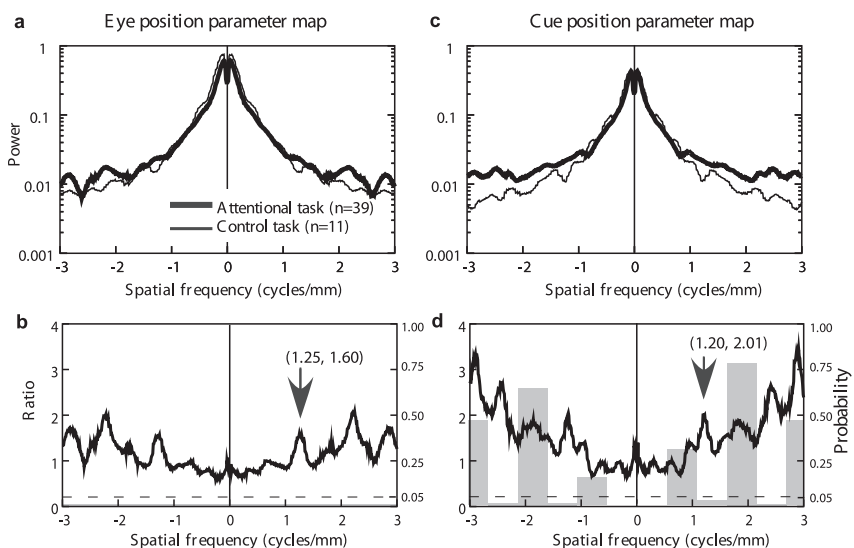

Figure 9. Average power of FFT across multiple experimental sessions for both animals. The RMS power for the spatial FFT was computed for each individual experimental case for the "up and down/ipsilateral and contralateral cue." This was performed for the attentional experiment and the control experiment. An FFT for the eye position parameter map and an FFT for the cue map were computed for each experiment after subtracting the average signal for the image. The FFTs were averaged and are plotted by the experimental conditions. The ratio of the attentional to the control experiments was also computed. $\boldsymbol{A}$, Average FFTs for the eye position maps. $\boldsymbol{B}$, The ratio for the eye position map. C, Average FFTs for the cue position maps. D, The ratio for the cue position maps. Each pair of curves was significantly different from each other using an ANOVA. $\boldsymbol{C}$ and $\boldsymbol{D}$ also depict probability estimates for the comparison of log power binned in 0.5 cycles $/ \mathrm{mm}$ in terms of the height of the gray bars. The dashed line indicates $p=0.05$. The left eye position panel has a very low-amplitude gray bar, indicating $p<0.001$, whereas the probability is more variable for the right cue position panel. Power is in units of percentage per degree per millimeter.

the two types of experiments. The majority of the experiments were performed with left-right attentional shifts $(n=39)$ and the left-right cue controls $(n=11)$, and so these were subject to additional quantitive analysis. The average brightness was subtracted from each parameter map for each experiment (DC removal), and then the FFT was computed. The average FFT was computed for each group of experiments across the two animals (Fig. 9). Two FFT curves were computed for each experiment in each animal: the first being for the eye position regression parameter, the second for the cue position. For both parameter maps, there is a divergence in the power at a spatial frequency of approximately \pm 1.2 and \pm 1.25 cycles $/ \mathrm{mm}$ for the two parameter maps. This corresponds to the characteristic spatial wavelength of $800-$ $833 \mu \mathrm{m}$. Similar results were found for each individual animal.

To quantitively compare the two conditions across the spatial frequencies, a two-way ANOVA was computed, with one independent variable being the spatial frequency and the other being the task condition (attentional shift vs control cue alone). These tests were performed for each animal individually (M1R and M2L) and for each map, resulting in four ANOVAs (Table 1). There were significant statistical differences between the attentional versus control conditions for every map for both animals. The significant effect of spatial frequency and task condition also was found when the data were combined across both animals. These graphical and statistical results demonstrate the scale of these patches being repeatable and significant across multiple experiments and two animals.

A second analysis was performed to determine which spatial frequencies were significantly different between the two conditions. The FFT power spectra were binned from -3 to $3 \mathrm{cy}-$ cles $/ \mathrm{mm}$ in 0.5 cycles $/ \mathrm{mm}$ bins. The logarithm of each value was computed, and a Student's $t$ test was used to compare the cued versus control conditions. As expected from the ANOVA, most of the comparisons were highly significant. Except for one set of conditions, the probability that the two conditions were significantly different was $p<0.001$. Even with an $\alpha$ correction for multiple comparisons, the cued and control power was different. The only case in which this was not true for all spatial frequencies was for the test when the monkey fixated up or down and the cue was provided left and right (as in Fig. 1a). Under these conditions, some of the bins were not significant for the cue-based map (Fig. 9B, right panel). These were between the peaks at 1.2 and 2.4 cycles $/ \mathrm{mm}$. The bins containing 1.2 and 2.4 cycles $/ \mathrm{mm}$ were significant.

The average spatial wavelength of $800 \mu \mathrm{m}$ across these experiments is similar to those obtained with almost identical quantitive FFT analysis of orientation tuning and ocular dominance from V1 optical data (Obermayer and Blasdel, 1993). A characteristic wavelength of $640-679 \mu \mathrm{m}$ and $822 \mu \mathrm{m}$ for orientation and ocular dominance, respectively, was reported. This suggests that similar neural mechanisms might subserve the scaling for the functional architecture of attentional patches in the inferior parietal lobule and that of primary visual cortex (Hubel and Wiesel, 1977). These results, taken with the monkeys' behavioral dependence on the cue location and the absence of cortical patchy modulation in the presence of the cue alone, lead to the conclusion that the emergence of the patches is correlated with the drawing of the monkey's spatial attention along the horizontal meridian.

\section{Stability and variability in maps}

It was evident from the segregation method on multiple data sets (data not shown) and visual inspection of the circle maps that there was variation in the distribution of the location of attentional patches across days. Although the position of the patches was variable, certain aspects of the maps were highly consistent across days and experimental conditions. First, the gain fields always showed a reliable and clear segregation at the blood vessel, which divided the imaged region into upper and lower eye position representations over the period March, 2000 to June, 2003 for M1R and an 8 month period for the second. Second, the periodicity of the patches was stable across the attentional experiments. Third, the patches were consistently absent when the monkeys' attention was restricted on the fixation point. Although there are examples of variability of representations across time (Wall et al., 1986; Kaas et al., 1990; Wilson and McNaughton, 1993; Darian-Smith and Gilbert, 1995), these can have very different underlying causes and timescales. The variability and stability of these measurements was examined in three ways.

\section{Monte Carlo analysis}

The intraday variability was examined in detail as an important constraint on the range of hypotheses and mechanisms for the interday variability of the location of the patches. First, a Monte Carlo analysis (Siegel et al., 2003) discounted stimulusindependent noise as the source of the patch structure on the data of an individual day (Fig. 10). Two randomized analyses were performed. First, half of the single trial images were randomly selected, and the maps were constructed. This process was repeated multiple times, and the results were averaged. The null hypothesis was that there was a random relationship between the images and the stimulus conditions. To construct data under this hypothesis, again, half of the trials were selected; however, they were randomly assigned to stimulus conditions. The average of 200 repetitions of this resulted in a flat map with no patterns. Hence, it was concluded that the patches could not arise randomly and were related to the location the animal was cued to attend. 
Spatial trial-by-trial analysis

A trial-by-trial analysis indicated that the patches varied as the experiment progressed, whereas the gain fields were fixed (Fig. 11). Patches could be observed in single trials (Fig. 11a,c); a mediolateral slice through each trial demonstrated that there was a sharp border in the gain fields, as reported previously (Siegel et al., 2003), at the central blood vessel, whereas the patch locations were not consistent from trial to trial (Fig. 11b). Other architectonic features in the control experiments remained constant across single trials (Fig. 11d).

To quantify these intraday effects, statistics were computed for the amplitude of this mediolateral slice by behavioral condition (Fig. 11e,f). The cued attention experiment showed periodicities in the amplitude of the signal (apparent by eye and quantified below), which were lacking in the control experiment. The control experiment presented a number of clearly demarcated transitions that could be observed in all classes. In contrast, the transitions between light and dark regions in the cued attention experiment were different across classes.

To compare the variability between the two experiments, the mean reflected light and SE was computed across all four behavioral conditions for both the control and attentional test (Fig. $11 e, f$; for clarity, the error bars are illustrated for only one behavioral condition per experimental test.) The SEs are clearly larger for the attentional test. To further quantify this, the average value of the SE across trials was then computed and compared for the control and cued experiment. The SE was $50 \%$ greater in the cued attention compared with the control test (i.e., $0.302 \pm 0.001$ vs $0.203 \pm 0.001 \%$, respectively; $n=943$ ). Thus, it is concluded that the spatial distribution of the reflected light has apparent periodicities (as expected from FFT of the parameter maps) and is more variable in the cued attention experiment compared with the control of attending to the fixation point.

\section{FFT trial-by-trial analysis}

A trial-by-trial analysis of the power of the frequency components demonstrated that the periodic patches were indeed present in each trial (Fig. 12). The spatial frequency from the patches of a single trial could be extracted (Fig. 12b). (For these measurements, the orientation of the FFT selected with the largest power was vertical in the image crossing the main blood vessel at $90^{\circ}$; see Materials and Methods.) Stacking these by condition (Fig. 12c) demonstrated an RMS power in the spatial frequency out to $\sim 1.5-2.0$ cycles $/ \mathrm{mm}$. It was not possible to discern a consistent relationship in the phase, even at $860 \mu \mathrm{m}$ across trials and conditions in the attentional task, suggesting that the patches were moving across the cortical surface (Fig. 12d). Certainly some phases were more common, otherwise the average spatial maps would be uniform. Stated another way, there were some portions of the cortex that tended to have similar signals and other that tended to be more variable.

The RMS power for the control experiment (Fig. 12g) had a narrow central peak that did not extend out to 1.5 cycles $/ \mathrm{mm}$. This indicated the lack of patches on a trial-by-trial basis. The consistency of the phase in the control experiment is as expected for the lower frequency components (Fig. 12h). To quantify the means and variability in the FFTs within trials, the data were converted from the polar-angular representation of RMS power and phase to complex rectangular coordinates for each single trial
FFT of Figure 12. To compute the variability of the power, the individual components in the complex plane for each frequency were averaged, and the mean and SE were computed. These provided both the average RMS power as a function of frequency, as well as the SE of the power measurement. Averaging in the complex plane ensures that the phase information is not lost and that nonlinearities in the power computation do not impact the estimated error.

The mean power spectra were similar across the four different classes of the cued attention task (Fig. 13a). The power spectra for the control task were also similar across the four stimulus conditions. Compared with the control task, the attentional power spectra was broad out to $1.5-2$ cycles $/ \mathrm{mm}$. To emphasize the regions that were different, the ratio of the two power spectra was computed for each of the four behavioral conditions (Fig. 13b). The spectra for all of these ratio plots peaked at $\sim 1.5$ cycles $/ \mathrm{mm}$, presumably representing the patches found in the cued attentional task and not the control task.

The mean phase profiles reflected the different characteristics of the data in the spatial domain (Fig. 13c,d). First, the mean phase as a function of frequency was different across the four behavioral conditions. This was observed for both the control and cued attention tasks and is equivalent to the differences found in the spatial domain. For example, in the control conditions, low $(<0.5$ cycles $/ \mathrm{mm})$ spatial frequency power is observed across all conditions (Fig. 13d). When the monkey is fixating down, and the cue is presented left and right, there is a phase shift at these low frequencies. This phase shift is essentially the area $7 \mathrm{a}$ and DP difference in signal from vertical eye position. When the animal is fixating up, the phase shift is reversed. These differences in the phase profiles in part underlie the low spatial frequency shifts in the average condition maps (Fig. 13f). For average condition maps, there is a vertical reversal in brightness between areas $7 \mathrm{a}$ and DP.

The phase plots are more complex in the cued attention task (Fig. 13c). Very little similarity occurs in the cue left versus right conditions when the monkey is fixating up, nor are the phase plots similar when the monkey looks down. Translating this result into the spatial domain, the variation in the mean phase reflects the different locations of the patches locations across the average condition map (Fig. 13e). Thus, for both the cued attention and the control task, the mean phase matches the spatial domain results.

The second characteristic of variability in the patches within a day is also reflected in the phase plots. Moreover, the use of the phase information permits isolation of the effects to the patch spatial frequency. The variability of the phase of the patches was examined within each stimulus condition (Fig. 14). A spatial fre- 
a. Eye position gain field component (up vs. down) Monte Carlo A
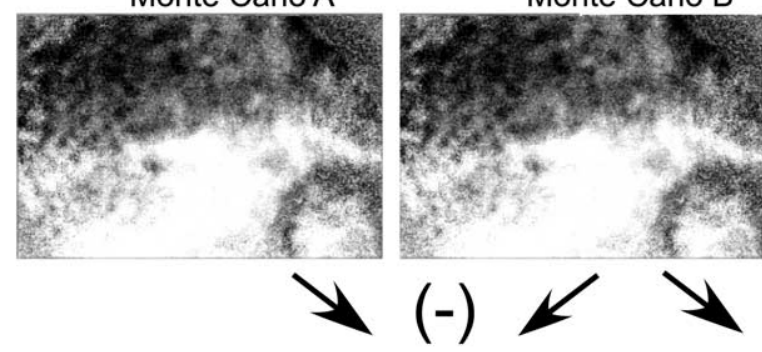

$\searrow(-)$
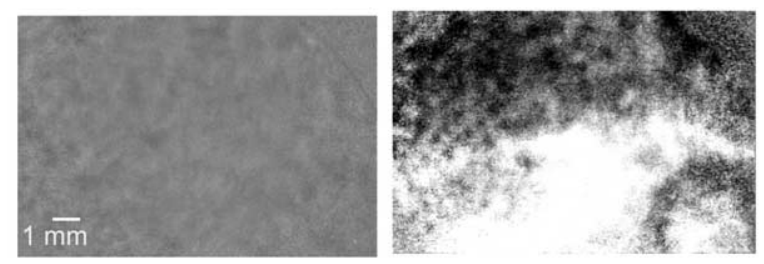

\section{b. Attentional cued component (left vs. right)}
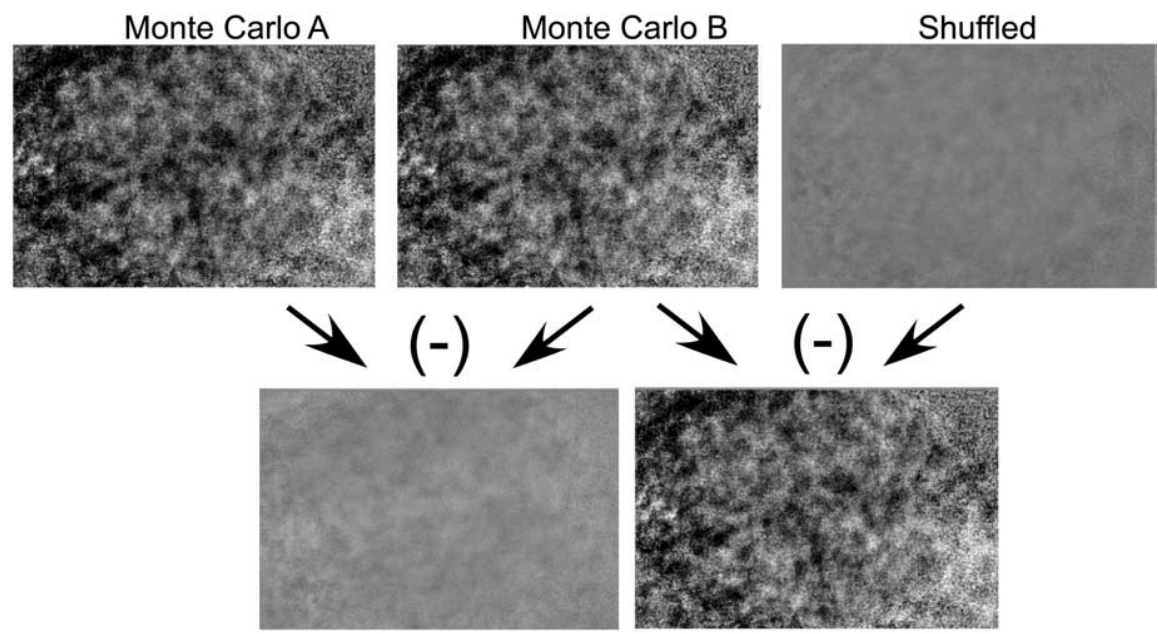

$(-)$

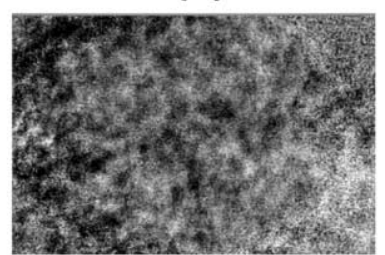

$-0.002 \% /$

$0.002 \% /^{\circ}$

Figure 10. Monte Carlo analysis of data from Figure 2 (M1R, 04-05-2001/gm). The Monte Carlo analysis was performed with and without shuffling the relationship between the measured optical signals and the stimulus conditions. Shuffling destroyed the patch structure. Hence, these patterns are dependent on the stimulus conditions. To show this, the trials from the experiments were randomly split into two subsets, and Equation 2 was fit to the data. The "Monte Carlo A" and "Monte Carlo B" maps are the resulting parameter maps. One-half of the data were also reanalyzed with Equation 2, but the relationship between the collected data and the stimulus condition was randomized, yielding the "Shuffled" maps. This process was repeated 200 times, yielding 600 maps. The averages of each of these groups are shown. $\boldsymbol{a}$, The eye position gain field parameter maps are reproducible when one-half of the data are sampled (Monte Carlo A and B). However, shuffling destroys the gain fields. The error between the Monte Carlo $A$ and $B$ is shown to be much less than that between Monte Carlo B and the Shuffled data. $\boldsymbol{b}$, The cue position parameter maps are reproducible. Shuffling the data destroys the cue position-related patches. Note that the gray scale on the images is different from other figures.

quency to represent the patches was selected to be centered at 1.5 cycles $/ \mathrm{mm}$ (1.25-1.75 cycles $/ \mathrm{mm}$ ) based on the power ratio (Fig. $13 b)$. The four distributions of phase angles were significantly different from each other using a distribution-free circular $\chi^{2}$ test $\left(p=0.004 ; \mathrm{df}=45 ;\right.$ bin width, $20^{\circ}$ ). To specifically examine the variation in the phase independent of the mean, the data are assumed to adhere to a von Mises distribution. This distribution is a circular equivalent of the normal distribution. The significance of difference in the variance of the angular phase (i.e., the concentration parameter) was computed by the WatsonWilliam-Mardia test (Batschelet, 1981). This measure of the 04-05-2001/gm

phase variability was significantly different $(p<0.001)$ across all four cued attention conditions $(p=0.003)$. These results indicate that ranges of phases at the spatial frequency of the patches were different across the different conditions. Translated to the spatial domain, these results indicate that the patches shift from trial to trial.

Together, the data and analyses of trialby-trial data support the hypothesis that the patches of $\sim 1.5$ cycles $/ \mathrm{mm}$ consistently appear during the cued attentional task and are lacking in the control task. These fixedsized patches are mobile. The phase of the patches is changing throughout the cued attentional task. The range of variability in phase is restricted enough so that the phases do not completely cancel each other in the course of an experiment (as might occur with totally random phases.)

\section{Upward versus downward cued locations}

The effect of upward and downward cues was also examined. In two monkeys, a set of experiments $(n=31)$ was performed with rightward and leftward fixations, and patchy maps were observed for upward and downward cue stimulus location (Figs. 15c, 16f). As expected from the previous study (Siegel et al., 2003), the horizontal gain fields maps (Figs. 15b, 16e) were much weaker than the vertical gain field. Using the circle space map, updown cued spatial location patches were observed to cover the cortical map in a manner similar to the left-right patches (Figs. 15e, 16g). Additional studies are needed to examine the precise relationship between the up-down and left-right patches in which monkeys have to shift their attention to one of four positions. Nonetheless, the complete coverage of the imaged portion of cortex for both vertical and horizontal loci of attention suggests a continuous and systematic millimeter scale representation of the direction of attention within the larger-scale gain fields; however, their precise organization remains unknown.

The patches were not observed when the cue-only physiological control was performed for cues in the upper and lower visual field $(n=9)$ (example in Fig. 16h-k). An analysis in two animals across this set of experiments demonstrated a 3.08-fold peak increase at 1.09 cycles/mm for the eye position map (wavelength of $917 \mu \mathrm{m}$ ). For the cue position maps, there was a 3.22-fold increase at 1.21 cycles $/ \mathrm{mm}(826 \mu \mathrm{m})$ in the power spectrum. The effect of spatial frequency and task was significant at the $p=0.0001$ level for both maps using an ANOVA. These wavelengths are similar to that of the left-right attention experiments.

One possibility for the variability in patch location (or phase) was that the patch location was determined by the monkey's 
behavioral performance. Although the monkey performed well above criterion in these experiments, there might have been a dependence on the reaction time. Hence, we ordered the frequency components and phase by the reaction time. There did not appear to be any consistent obvious variation in the spatial frequency or phase with the reaction time (data not illustrated). An additional alternative was that the spatial frequency and phase changed in an orderly manner through the experiment; ordering the data by the trial sequence also did not reveal an effect. However, more quantitive and higher dimensional approaches such as independent component analysis currently under development might reveal the source of the variation of the phase of the patches as relates the monkeys' measurable behavior (Siegel et al., 2002).

\section{Discussion}

\section{Behavioral evidence for shifts in spatial attention}

Attention was directed spatially using a task based on Posner's (1980) original behavioral paradigm. The monkeys demonstrated a shift of attention, as evidenced by the reaction-time dependence on cue location and validity. If the animals had simply ignored the cued location, distributing their attention evenly across the screen, or rapidly shifting their attention back and forth, then the reaction times should not have been dependent on the cue location and validity.

\section{Characteristics of the gain field and attentional topographies}

The optical data collected during the task was modeled as separable eye position-dependent and cue position-dependent components. The eye position parameter map had two spatial components: a portion divided between areas $7 \mathrm{a}$ and DP and the $\sim 1 \mathrm{~mm}$ patches. The component of the eye position was split between areas $7 \mathrm{a}$ and DP, matching that described for the gain fields in our previous work (Siegel et al., 2003). The cue position map only had the $\sim 1 \mathrm{~mm}$ patches that ran across the $7 \mathrm{a} / \mathrm{DP}$ border. The border in the eye position map between areas $7 \mathrm{a}$ and DP was stable both within and across experiments. The location of the patches in the combined color-coded parameter maps shifted between experimental days. This was evident in shifts in phase in the Fourier-transformed data of the parameter maps. The invariant size of the patches was evident from the spatial frequency of the patches.

\section{Concomitant stability of eye position and variability of the patches}

Functional architectures are typically temporally stable when assessed with optical imaging. The primary evidence is orientation tuning and ocular dominance in striate cortex of behaving monkey. However, in most intrinsic optical imaging studies, the animal is anesthetized and its cognitive state (e.g., attention and intention) is irrelevant. In the striate cortex studies in behaving monkey for which anesthesia was not used (Grinvald et al., 1991; Vnek et al., 1999;
Shtoyerman et al., 2000; Arieli et al., 2002), the monkey was typically required to fixate a central point and not shift its attention from that location. Such stability in striate obtained with intrinsic imaging indicates that the technique itself is reliable. Indeed, in our experimental setup, a stable retinotopy across multiple days in V1 (Heider et al., 2005) and a stable representation of eye position in areas $7 \mathrm{a}$ and DP over months (Siegel et al., 2003) is demonstrated.

Not all cortical representations must be completely stable over time, especially when modulated by cognitive factors. The retinotopy studies of the inferior parietal lobule suggest that there can be variability in its representation (Heider et al., 2005). Indeed, studies of plasticity in the somatosensory cortex (Allard et al., 1991) and in primary visual cortex (Kaas et al., 1990; DarianSmith and Gilbert, 1995) make it clear that maps are malleable. Gilbert (1993) has made an explicit proposal that functional architectures were plastic. Additional perceptual learning alters the tuning properties of single neurons in V4 (Yang and Maunsell, 2004). Given that many neurons will be involved, the map across V4 must change. In other studies in which the receptive field properties of neurons in parietal cortex are altered on a trial-bytrial basis (Bushnell et al., 1981; Constantinidis and Steinmetz, 2001), the emergent map must also change.

In the present studies, some aspects of the inferior parietal lobule functional architecture were variable across time, and some were unchanging. The locations of the patches changed across days and even within sessions. However, at the same time 


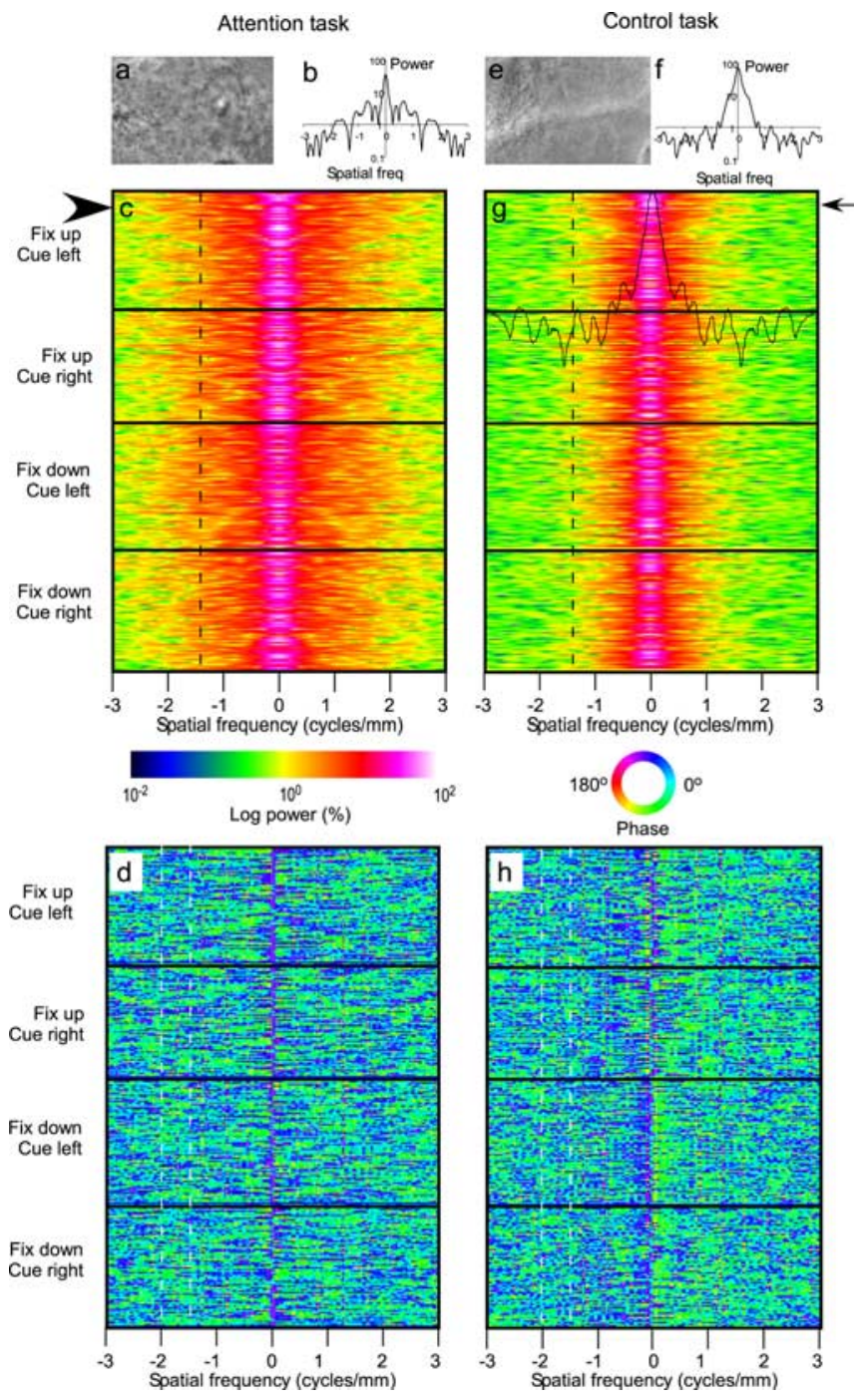

Figure 12. Trial-by-trial analysis of the frequency components for the spatial attention experiment and the control. Each trial was analyzed using an FFT as described for Figure 11. The resulting FFTs were color coded and organized by stimulus condition and by stimulus presentation. $\boldsymbol{a}, A$ single trial in the spatial domain collected when the monkey was fixating up and the cue directed attention to the left. The periodic patches can be seen in this trial (and indeed in every trial of this experiment). frea, Frequency. $\boldsymbol{b}$, FFT of the single trial with peaks in the power spectrum. $\boldsymbol{c}$, The stacked FFT power of all of the trials. The arrow to the left indicates the FFT computed in $\boldsymbol{b}$. The phase appears random from trial to trial. Fix, Fixation. $\boldsymbol{d}$, Control task circle map. $\boldsymbol{e}$, A single trial taken when the monkey was fixating up, attending to the fixation point; the "ignored" cue was to the left. Patches could not be observed in the individual trials of this experiment. $\boldsymbol{f}$, FFT of the single trial. Periodicities can be observed, but they are smaller in amplitude. $\boldsymbol{g}$, Stacked FFTs for the control task. There is no periodicity apparent at the 1 cycle/mm value in the trials. $\boldsymbol{h}$, The phase is slightly, although consistently, retarded for frequencies $<1$ cycle $/ \mathrm{mm}$

this was occurring, the eye position gain field representation was stable. The internal control of stability of the eye position signal indicates that the recordings themselves have stability. What is perhaps even more intriguing is that, although patch locations were variable across and within days (as evidenced by direct inspection and by the spatial phase), the spatial frequency map was temporally stable. These data indicate multiple coactive mechanisms in areas $7 \mathrm{a}$ and DP simultaneously generating different types of cortical representations.

\section{Source of the variability in the cued attentional task}

The question arises as to the source of the variability in the cued attentional task. The variability could arise from instrumentation noise. However, recordings in V1 made with the identical system show no such variability (Heider et al., 2005). Furthermore, the measurements were made with different magnifications. Any sensor noise would have lead to the patches size being insensitive to magnification.

A second source for the variable topography of the attentional maps could arise from changes in the monkey's behavioral strategy. For example, there could be small shifts of attention within the peripheral visual field to which the monkey directed his attention. Indeed, the monkey could set his attention anywhere within the $10^{\circ}$ optic flow stimulus. Additional experiments are needed to explore this possibility, including shrinking the size of the stimulus to tightly restricting the locus of the monkeys' attention. Indeed, alterations in attentional strategy have been posited as the source of variability in retinotopic maps in inferior parietal lobule (Heider et al., 2005).

A third major source of the changes in the maps is physiological variation or physiological noise. For example, it is well accepted that there is variation of neuronal discharge from unknown sources (Shadlen and Newsome, 1998). Furthermore, the coupling between the neuronal activity and the hemoglobin signal could vary as a result of physiological factors beyond experimental control (e.g., heart or respiration rate) (Logothetis, 2002). Finally, the variability could arise directly from dynamic cortical processing strategies (Edelman, 1987; Crick and Koch, 2003). With each successive stimulus presentation and behavioral trial, different collections of neurons may be activated through the interactions between ascending, descending, and intracortical circuits. Indeed, such malleable neural circuit dynamics could arise from, or be the source of, different behavioral strategies.

At this time, it is impossible to resolve which of these behavioral or physiological sources could account for the variability in the attentional patches reported here. Two types of experiments can further explore these possibilities. First, manipulations of the experimental design to alter the vigilance and attentional focus of the animal may be used to determine whether the behavioral variability may be placed under experimental control. This would separate nonspecific physiological effects from sources directly related to aspects of the attentional task. The second series of experiments would be measurements of single neurons through electrical means. Certain predictions are next.

\section{Expected electrical measurements}

Intrinsic optical signals are indirect measurements of overall neuronal activity arising from metabolic activity in the upper cortical layers. However, in every study performed to date, there has been a match between the neuronal properties evaluated using singleelectrode electrical measurements and optical measurements, including areas 7a and DP (Siegel et al., 2003). These intrinsic signals are related to the fine-scale axonal and dendritic elements with large surface area to volume ratio (Malonek and Grinvald, 1996; Logothetis, 2003). Thus, it is predicted that recordings from multiple electrodes should show variable tuning in either the local field potential or single-unit activity over time. The observed variation in phase should be observable if long-term chronic recordings are made from multiple sites.

Recordings from collections of neurons could be used to test whether Crick and Koch's (2003) hypothesis of dynamical cortical processing is expressed in the activity of inferior parietal lobule neurons. Depending on the exact inputs and interactions of 
a) RMS Power
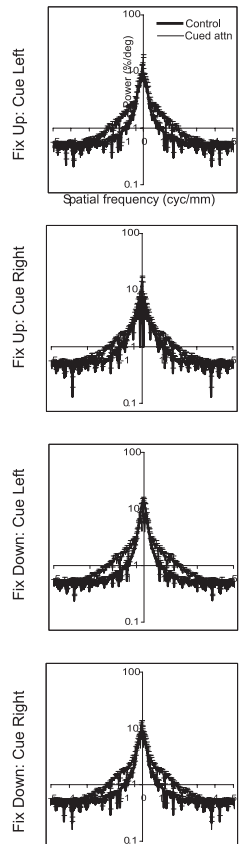

b) Power Ratio
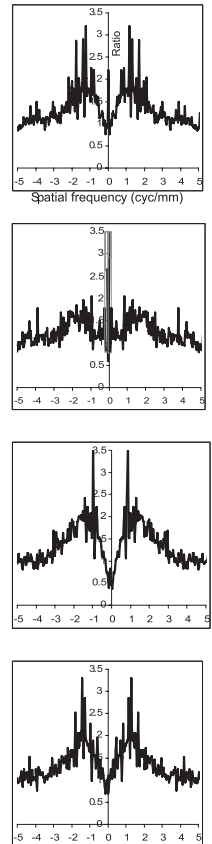

c) Phase: cued attention

d) Phase: control
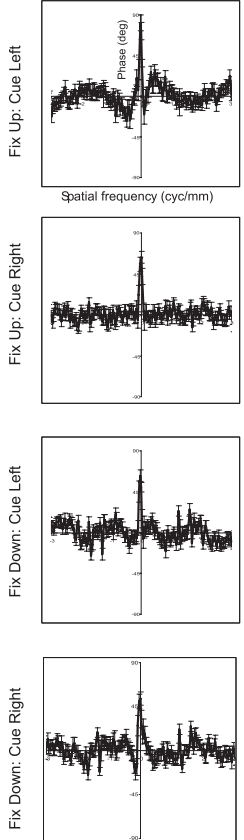
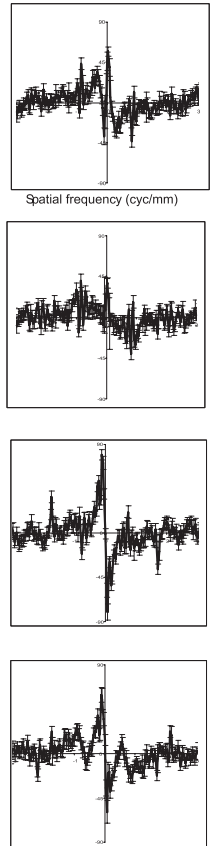

e) Spatial: cued attention
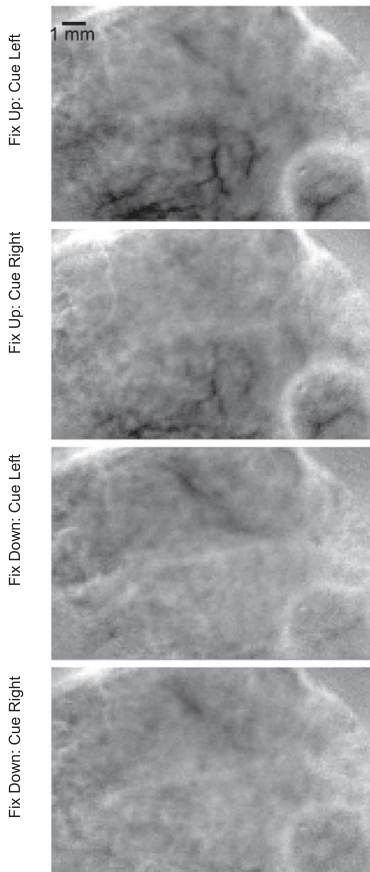

f) Spatial: contro
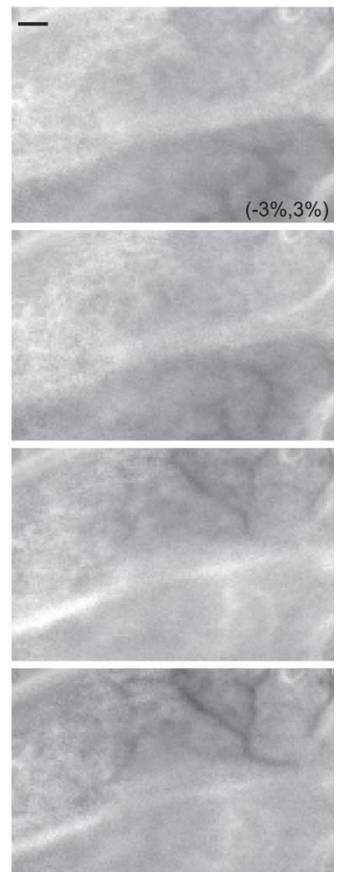

Figure 13. Quantitive evaluation of the mean and error of the Fourier-transformed single-condition maps. $\boldsymbol{a}$, The average and SE of the RMS power were computed from the FFTs of Figure 12 as described in Results. The SE of the power is illustrated in one direction. [The repeated downward deflections in the control graphs arise from the repeated sharp valleys in the individual FFTs (Fig. 12F).] The RMS power of the cued attentional task is broader than that of the control task. Fix, Fixation. $\boldsymbol{b}$, Ratio of the power in the attentional and control tasks. The power is approximately threefold greater for the attentional task than the control, peaking at $\sim 1.5$ cycles $/ \mathrm{mm}$. The spikes in the ratio plots arise from the repeated sharp valleys of the mean control power. $c$, Circular mean and SEM for the phase for the cued attention task (for computational details, see Results). $\boldsymbol{d}$, Circular mean and SEM for the control task. $\boldsymbol{e}$, Single-condition maps for cued attention task. $\boldsymbol{f}$, Single-condition maps for the control task. Data are from experiments of 04-05-2001/gm and 10-05-2001/gm. Scale bars, $1 \mathrm{~mm}$.

\section{Attentional task: Phase at $1.5 \mathrm{cyc} / \mathrm{mm}$ \\ Cue Left}
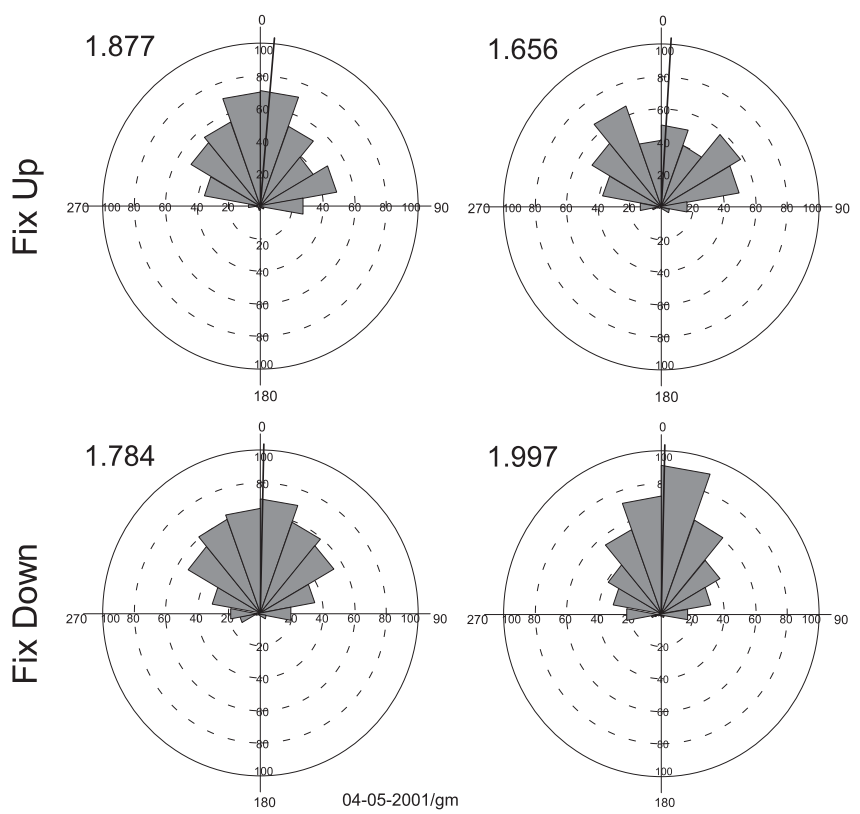

Figure 14. Distribution of the trial-by-trial phase angles evaluated at 1.5 cycles $/ \mathrm{mm}$ for the cued attention task. The location of each rose plot indicates the behavioral condition. The circular mean phase is indicated by the lines near $0^{\circ}$. The concentration assuming a von Mises distribution is indicated to the top left of each rose plot. Higher values of the concentration indicate a more compact collection of phases. Histograms were computed using $20^{\circ}$ bins. The concentrations of the four distributions were significantly different from each other (see Results). Fix, Fixation.

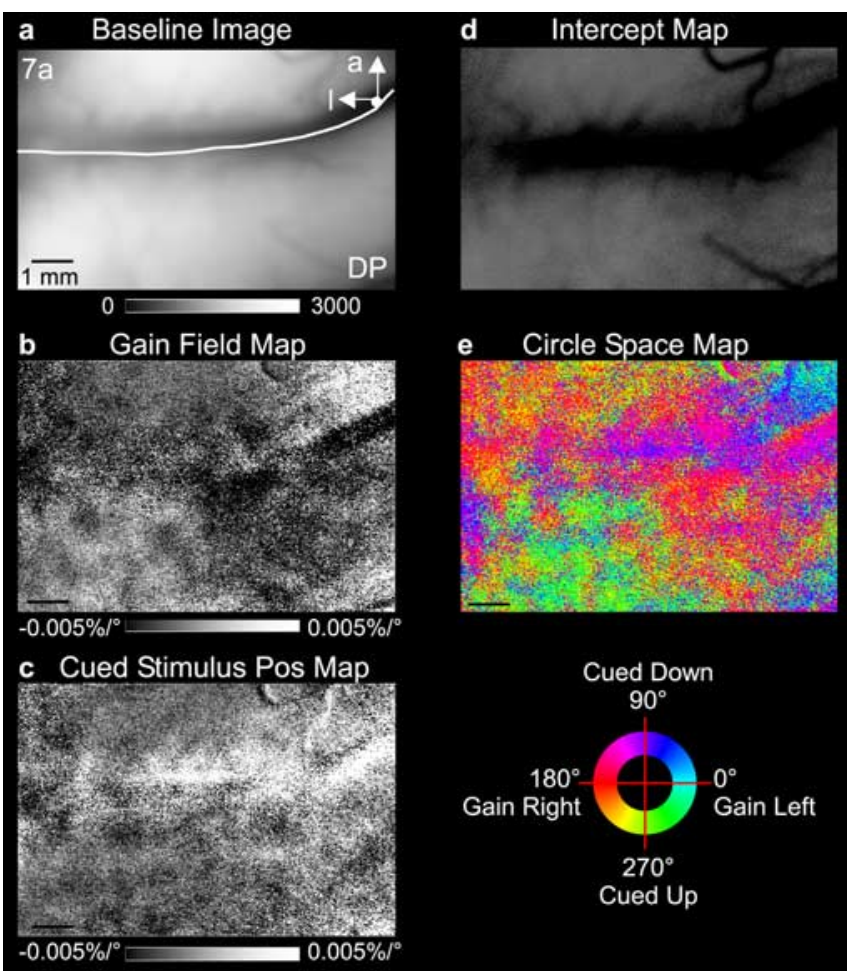

Figure 15. Cortical topography for spatial attention in the left inferior parietal lobule of M2L with eye position shifts left and right and cued stimulus position up and down. $\boldsymbol{a}$, Image of areas $7 \mathrm{a}$ and DP taken with $605 \mathrm{~nm}$ illumination. The white line shows the putative border between the two areas. Linear regression analysis. $\boldsymbol{b}$, Gain field map for horizontal eye position. $\boldsymbol{c}$, Cued stimulus location map for vertical positions. Pos, Position. $\boldsymbol{d}$, The intercept map reveals the underlying vasculature. $\boldsymbol{e}$, Circle space map. a, Anterior; l, lateral. Data are from 01-29-2003/gm. Scale bars, $1 \mathrm{~mm}$. 

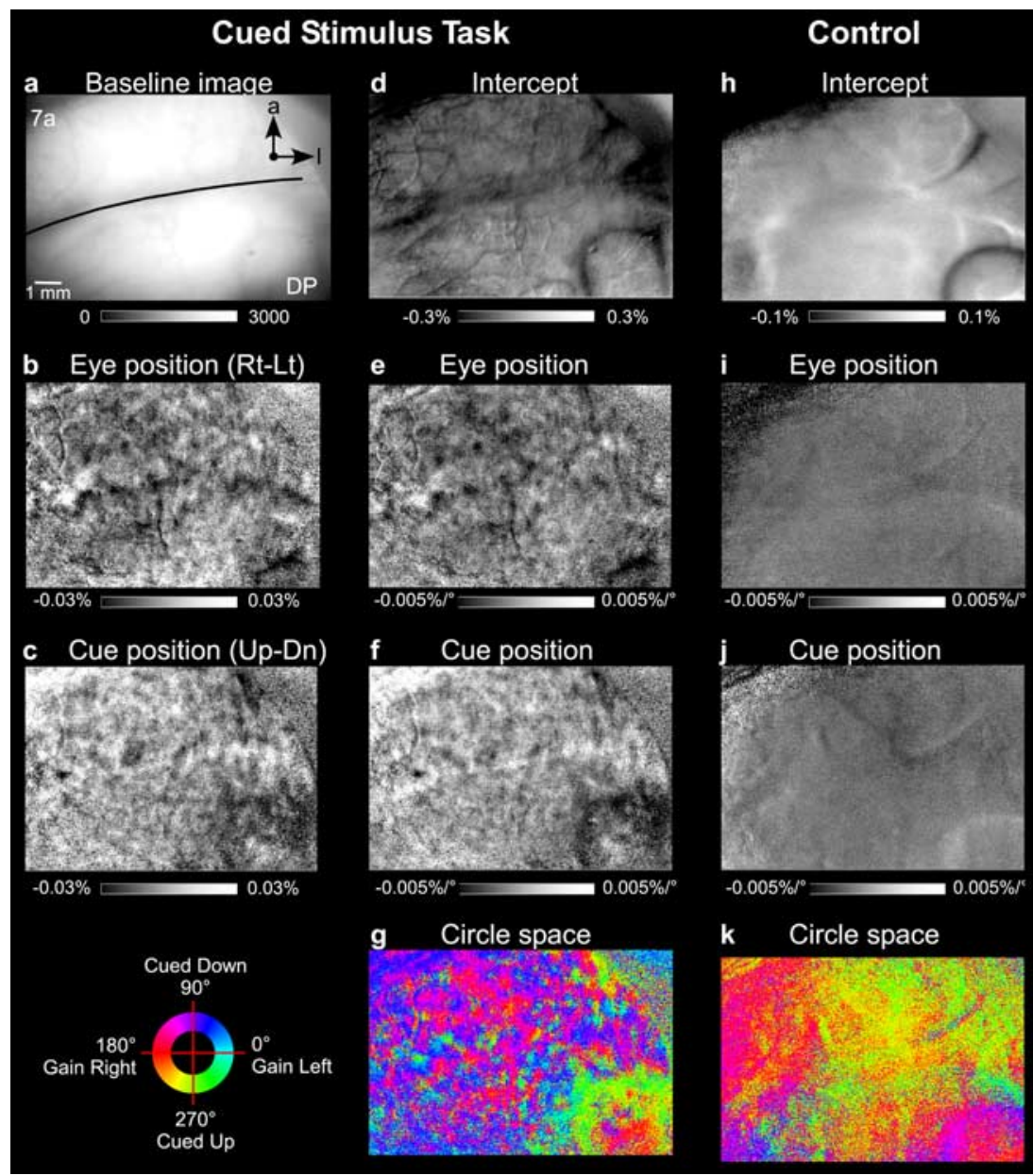

$\mathbf{k}$

Circle space

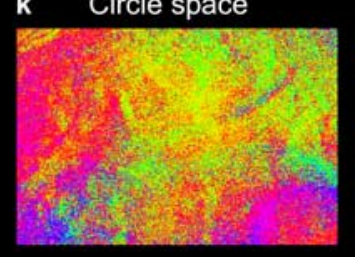

Figure 16. Cortical topography for spatial attention in the inferior parietal lobule (M1R) with eye position shifts left and right and cued stimulus position up and down. Conventions are as in Figure 2. $\boldsymbol{a}$, Average reflected light across the cortex imaged with red (605 nm) illumination. $\boldsymbol{b}$, Subtraction analysis for eye position map; all rightward (Rt) fixation conditions were averaged and subtracted from all leftward (Lt) fixation conditions. c, Subtraction cued stimulus position map; upward (Up) cued conditions were subtracted from downward (Dn) cued conditions. $\boldsymbol{d}$, Intercept of the regression. $\boldsymbol{e}$, Gain field parameter map. $\boldsymbol{f}$, Cued stimulus position parameter map. $\boldsymbol{g}$, Circle space map of direction of steepest slope. Pure gain fields are represented by light blue and red; deviations from these indicate the effect of the cued stimulus position. $\boldsymbol{h}-\boldsymbol{k}$, Physiological controls analyzed by linear regression model. $\boldsymbol{h}$, Intercept. $\boldsymbol{i}$, Gain field map. $\boldsymbol{j}$, Cued stimulus position map. $\boldsymbol{k}$, Circle space map. All data are from animal M1R $(\boldsymbol{a}-\boldsymbol{g}$, 04-11-2001/gm; $\boldsymbol{h}-\boldsymbol{k}$, 04-29-2002/gm). a, Anterior; l, lateral. Chamber edge, top right.

inferior parietal lobule, different "nascent coalitions" of neurons may dominate over other collections of neurons, giving rise to the spatial variability we report.

Electrode recordings were not performed here because of the need to maintain the imaging chambers for many years (Siegel et al., 2003). The electrode makes holes in the artificial dura, and, when it reaches the fine microgranular layer of tissue formed between the cortex and the artificial dura, it can catch and either tear the tissue or substantially indent the cortex, compromising the vasculature and jeopardizing the longevity of the study. Different technical approaches need to be developed to permit simultaneous long-term assessment of local field potentials, single units, and optical measurements to test these varied hypotheses.

\section{The role of a variable functional architecture}

The fine attentional architecture is embedded within the largerscale gain field topography, much as orientation tuning is mapped within retinotopy in primary visual cortex. The systematic repetition of patches embedded in linear gain fields guarantees that, for every combination of eye position and attentional locus, patches throughout areas 7a and DP will be modulated (Siegel, 1998). This is unlike more sensory-based topographies, e.g., V1 (Hubel and Wiesel, 1977; Ts'o et al., 1990; Blasdel and Campbell, 2001) or the middle temporal area (MT/V5) (Albright et al., 1984), which are locally organized around spatially delimited receptive fields. If the variability in the patches indeed represents a variable mapping superimposed on a highly consistent gain field, then a mechanism for areas $7 \mathrm{a}$ and DP to participate in diverse functions emerges. It has not escaped our notice that the on-line neural construction of the patches would permit this portion of association cortex to subserve a multitude of cognitive processes, as a function of the ongoing inputs and behavioral state. However, a "readout" mechanism for such a variable map remains to be defined.

The result of a distributed spatial attention representation within the large-scale gain field topography should allow for a multitude of local interactions among nearby attentional patches. The lateral excitatory and inhibitory interactions of the upper layers (Callaway, 1998) should support the formation and dissolution of particular coalitions of neurons (Crick and Koch, 2003) either correlated with, or causing, shifts of attention. This is a different mechanism than the attentional spotlight model (Crick, 1984), although these patches could be under thalamic (i.e., pulvinar) control. The interrelationships between eye position gain fields and the attentional representations would be ideal for guiding and planning eye movements and other motor behaviors toward particularly salient targets in the surrounding world (Goodale and Milner, 1992; Siegel, 1998).

Two different hypothetical expressions of functional architectures may be involved here. The first is rather well established and accepted: "topography reflects anatomical connectivity." An example is ocular dominance in adult Old World primates. Projections from the lateral geniculate nucleus are segregated, forming the ocular dominance patterns that can be imaged by 2-deoxyglucose studies, single-unit recordings, or optical imaging. We term this the "anatomical hypothesis" for cortical topography.

The second hypothesis is based on a different set of considerations: "ongoing neuronal activity is the source of the topographic map." Maps are based, in part, on the existing connectivity, but modulation, activation, depression of synapses, interactions between neighboring neurons, etc. can alter the functional architecture of the relationships between neurons across the cortex "on-line" over rapid timescales. There are a number of examples of altered primary sensory topography by external manipulations (Jenkins et al., 1990; Kaas et al., 1990; Allard et al., 1991; Darian-Smith and Gilbert, 1995; Li et al., 2004), but these studies lacked the means to appreciate how quickly the maps could change. The topography could be altered across the second timescale, as appears to occur in inferior pari- 
etal lobule under cognitive control. Ultimately, both the "anatomical" and functional mechanisms will play a role in normal brain function and in the plasticity of the brain in individuals who suffer neurological compromise.

\section{References}

Akaike H (1969) Fitting autoregressive models for prediction. Ann Inst Statistical Math 21:243-247.

Albright TD, Desimone R, Gross CG (1984) Columnar organization of directionally selective cells in visual area MT of the macaque. J Neurophysiol 51:16-31.

Allard T, Clark SA, Jenkins WM, Merzenich MM (1991) Reorganization of somatosensory area $3 \mathrm{~b}$ representations in adult owl monkeys after digital syndactyly. J Neurophysiol 66:1048-1058.

Andersen RA, Essick GK, Siegel RM (1985) The encoding of spatial location by posterior parietal neurons. Science 230:456-458.

Andersen RA, Asanuma C, Essick G, Siegel RM (1990) Corticocortical connections of anatomically and physiologically defined subdivisions within the inferior parietal lobule. J Comp Neurol 296:65-113.

Arieli A, Grinvald A, Slovin H (2002) Dural substitute for long-term imaging of cortical activity in behaving monkeys and its clinical implications. J Neurosci Methods 114:119-133.

Astafiev SV, Shulman GL, Stanley CM, Snyder AZ, Van Essen DC, Corbetta M (2003) Functional organization of human intraparietal and frontal cortex for attending, looking, and pointing. J Neurosci 23:4689-4699.

Batschelet E (1981) Circular statistics in biology (mathematics in biology). London: Academic.

Blasdel G, Campbell D (2001) Functional retinotopy of monkey visual cortex. J Neurosci 21:8286-8301.

Bushnell MC, Goldberg ME, Robinson DL (1981) Behavioral enhancement of visual responses in monkey cerebral cortex. I. Modulation in posterior parietal cortex related to selective visual attention. J Neurophysiol 46:755-772.

Callaway EM (1998) Local circuits in primary visual cortex of the macaque monkey. Annu Rev Neurosci 21:47-74.

Constantinidis C, Steinmetz MA (2001) Neuronal responses in area 7a to multiple-stimulus displays. I. Neurons encode the location of the salient stimulus. Cereb Cortex 11:581-591.

Crick F (1984) Function of the thalamic reticular complex: the searchlight hypothesis. Proc Natl Acad Sci USA 81:4586-4590.

Crick F, Koch C (2003) A framework for consciousness. Nat Neurosci 6:119-126.

Critchley M (1953) The parietal lobes. New York: Hafner.

Darian-Smith C, Gilbert CD (1995) Topographic reorganization in the striate cortex of the adult cat and monkey is cortically mediated. J Neurosci 15:1631-1647.

Edelman G (1987) Neural darwinism: the theory of neuronal group selection. New York: Basic Books.

Gilbert CD (1993) Rapid dynamic changes in adult cerebral cortex. Curr Opin Neurobiol 3:100-103.

Goodale MA, Milner AD (1992) Separate visual pathways for perception and action. Trends Neurosci 15:20-25.

Grinvald A, Frostig RD, Siegel RM, Bartfeld E (1991) High-resolution optical imaging of functional brain architecture in the awake monkey. Proc Natl Acad Sci USA 88:11559-11563.

Heider B, Jando G, Siegel RM (2005) Functional architecture of retinotopy in visual association cortex of behaving monkey. Cereb Cortex 15:460-478.

Heilman KM, Watson RT, Valenstein E (1993) Neglect and related disorders. In: Clinical neuropsychology, Ed 3 (Heilman KM, Valenstein E, eds), pp 279-336. New York: Oxford UP.

Hubel DH, Wiesel TN (1977) Ferrier lecture. Functional architecture of macaque monkey visual cortex. Proc R Soc Lond B Biol Sci 198:1-59.

Jenkins WM, Merzenich MM, Ochs MT, Allard T, Guic-Robles E (1990) Functional reorganization of primary somatosensory cortex in adult owl monkeys after behaviorally controlled tactile stimulation. J Neurophysiol 63:82-104.

Kaas JH, Krubitzer LA, Chino YM, Langston AL, Polley EH, Blair N (1990) Reorganization of retinotopic cortical maps in adult mammals after lesions of the retina. Science 248:229-231.

Kastner S, Ungerleider LG (2000) Mechanisms of visual attention in the human cortex. Annu Rev Neurosci 23:315-341.

Li W, Piëch v, Gilbert CD (2004) Perceptual learning and top-down influences in primary visual cortex. Nat Neurosci 7:651-657.
Logothetis NK (2002) The neural basis of the blood-oxygen-leveldependent functional magnetic resonance imaging signal. Philos Trans $\mathrm{R}$ Soc Lond B Biol Sci 357:1003-1037.

Logothetis NK (2003) The underpinnings of the BOLD functional magnetic resonance imaging signal. J Neurosci 23:3963-3971.

Malonek D, Grinvald A (1996) Interactions between electrical activity and cortical microcirculation revealed by imaging spectroscopy: implications for functional brain mapping. Science 272:551-554.

Motter BC, Mountcastle VB (1981) The functional properties of the lightsensitive neurons of the posterior parietal cortex studied in waking monkeys: foveal sparing and opponent vector organization. J Neurosci 1:3-26.

Mountcastle VB, Andersen RA, Motter BC (1981) The influence of attentive fixation upon the excitability of the light-sensitive neurons of the posterior parietal cortex. J Neurosci 1:1218-1225.

Nadeau SE, Heilman KM (1991) Gaze-dependent hemianopia without hemispatial neglect. Neurology 41:1244-1250.

Nadeau SE, Crosson B, Schwartz RL, Heilman KM (1997) Gaze related enhancement of hemispheric blood flow in a stroke patient. J Neurol Neurosurg Psychiatry 62:538-540.

Newsome WT, Wurtz RH, Dursteler MR, Mikami A (1985) Deficits in visual motion processing following ibotenic acid lesions of the middle temporal visual area of the macaque monkey. J Neurosci 5:825-840.

Obermayer K, Blasdel GG (1993) Geometry of orientation and ocular dominance columns in monkey striate cortex. J Neurosci 13:4114-4129.

Posner MI (1980) Orienting of attention. Q J Exp Psychol 32:3-25.

Posner MI, Petersen SE (1990) The attention system of the human brain. Annu Rev Neurosci 13:25-42.

Posner MI, Walker JA, Friedrich FJ, Rafal RD (1984) Effects of parietal injury on covert orienting of attention. J Neurosci 4:1863-1874.

Rafal RD (1994) Neglect. Curr Opin Neurobiol 4:231-236.

Read HL, Siegel RM (1997) Modulation of responses to optic flow in area 7a by retinotopic and oculomotor cues in monkey. Cereb Cortex 7:647-661.

Schiller PH, Tehovnik EJ (2003) Cortical inhibitory circuits in eyemovement generation. Eur J Neurosci 18:3127-3133.

Shadlen MN, Newsome WT (1998) The variable discharge of cortical neurons: implications for connectivity, computation, and information coding. J Neurosci 18:3870-3896.

Shtoyerman E, Arieli A, Slovin H, Vanzetta I, Grinvald A (2000) Long-term optical imaging and spectroscopy reveal mechanisms underlying the intrinsic signal and stability of cortical maps in V1 of behaving monkeys. J Neurosci 20:8111-8121.

Siegel RM (1998) Representation of visual space in area 7a neurons using the center of mass equation. J Comput Neurosci 5:365-381.

Siegel RM, Andersen RA (1988) Perception of three-dimensional structure from two-dimensional motion in monkey and man. Nature 3319:259-261.

Siegel RM, Read HL (1997) Analysis of optic flow in the monkey parietal area 7a. Cereb Cortex 7:327-346.

Siegel RM, Duann JR, Jung TP, Sejnowski TJ (2002) Independent component analysis of intrinsic optical signals for gain fields in inferior parietal cortex of behaving monkey. Soc Neurosci Abstr 28:622.2.

Siegel RM, Raffi M, Phinney RE, Turner JA, Jando G (2003) Functional architecture of eye position gain fields in visual association cortex of behaving monkey. J Neurophysiol 90:1279-1294.

Taylor TL, Klein RM (2000) Visual and motor effects in inhibition of return. J Exp Psychol Hum Percept Perform 26:1639-1656.

Ts'o DY, Frostig RD, Lieke EE, Grinvald A (1990) Functional organization of primate visual cortex revealed by high resolution optical imaging. Science 249:417-420.

Vnek N, Ramsden BM, Hung CP, Goldman-Rakic PS, Roe AW (1999) Optical imaging of functional domains in the cortex of the awake and behaving monkey. Proc Natl Acad Sci USA 96:4057-4060.

Wall JT, Kaas JH, Sur M, Nelson RJ, Felleman DJ, Merzenich MM (1986) Functional reorganization in somatosensory cortical areas $3 \mathrm{~b}$ and 1 of adult monkeys after. J Neurosci 6:218-233.

Wardak C, Olivier E, Duhamel JR (2002) Saccadic target selection deficits after lateral intraparietal area inactivation in monkeys. J Neurosci 22:9877-9884.

Wilson MA, McNaughton BL (1993) Dynamics of the hippocampal ensemble code for space. Science 261:1055-1058.

Yang T, Maunsell JH (2004) The effect of perceptual learning on neuronal responses in monkey visual area V4. J Neurosci 24:1617-1626. 